\title{
Detection of knots and a cabling formula for A-polynomials
}

\author{
$\mathrm{Yi} \mathrm{Ni}$ \\ Department of Mathematics, Caltech \\ 1200 E California Blvd, Pasadena, CA 91125 \\ Email: yini@caltech.edu \\ Xingru Zhang \\ Department of Mathematics, University at Buffalo \\ Email: xinzhang@buffalo.edu
}

\begin{abstract}
We say that a given knot $J \subset S^{3}$ is detected by its knot Floer homology and $A$-polynomial if whenever a knot $K \subset S^{3}$ has the same knot Floer homology and the same $A$-polynomial as $J$, then $K=J$. In this paper we show that every torus knot $T(p, q)$ is detected by its knot Floer homology and $A$-polynomial. We also give a one-parameter family of infinitely many hyperbolic knots in $S^{3}$ each of which is detected by its knot Floer homology and $A$-polynomial. In addition we give a cabling formula for the A-polynomials of cabled knots in $S^{3}$, which is of independent interest. In particular we give explicitly the A-polynomials of iterated torus knots.
\end{abstract}

\section{Introduction}

One of basic problems in knot theory is to distinguish knots in $S^{3}$ from each other using knot invariants. There are several knot invariants each being powerful enough to determine if a given $\operatorname{knot}$ in $S^{3}$ is the unknot, such as the knot Floer homology [25, the A-polynomial [3] 11], and the Khovanov homology 20]. In other words, these invariants are each an unknot-detector. It is also known that the knot Floer homology can detect the trefoil knot and the figure 8 knot [14. In this paper we first consider the problem of detecting the set of torus knots $T(p, q)$ in $S^{3}$ using knot invariants. To reach this goal either the knot Floer homology or the $A$-polynomial alone is not enough; for instances the torus knot $T(4,3)$ has the same knot Floer homology as the $(2,3)$-cable over $T(3,2)[18$, and the torus knot $T(15,7)$ has the same $A$-polynomial as the torus knot $T(35,3)$. However when the two invariants are combined together, the job can be done. We have

Theorem 1.1. If a knot $K$ in $S^{3}$ has the same knot Floer homology and the same A-polynomial as a torus knot $T(p, q)$, then $K=T(p, q)$.

We then go further to find out a one-parameter family of mutually distinct hyperbolic knots $k\left(l_{*},-1,0,0\right)$ in $S^{3}$, where $l_{*}>1$ is integer valued, each of which is detected by the combination of its $A$-polynomial and its knot Floer homology. A knot diagram for $k\left(l_{*},-1,0,0\right)$ is illustrated in Figure 5 . Note that $k(2,-1,0,0)$ is the $(-2,3,7)$-pretzel knot. Also note that $k\left(l_{*},-1,0,0\right)$ is a subfamily of the hyperbolic knots $k(l, m, n, p)$ (with some forbidden values on integers $l, m, n, p)$ given in [12] each of which admits a half-integral toroidal surgery (the slope formula is given in [13, and recalled in Section 4 of this paper) and by [17] these hyperbolic knots $k(l, m, n, p)$ are the only hyperbolic knots in $S^{3}$ which admit non-integral toroidal surgeries.

Theorem 1.2. The family of knots $\left\{k\left(l_{*},-1,0,0\right) ; l_{*}>1, l_{*} \in \mathbb{Z}\right\}$ are mutually distinct hyperbolic knots in $S^{3}$. Let $J_{*}$ be any fixed $k\left(l_{*},-1,0,0\right), l_{*}>1$. If a knot $K$ in $S^{3}$ has the same knot Floer homology and the same A-polynomial as $J_{*}$, then $K=J_{*}$. 
The key input from the knot Floer homology in proving Theorems 1.1 and 1.2 is that $K$ is fibred [14, 23] (since $T(p, q)$ or $J_{*}$ is) and has the same Alexander polynomial as $T(p, q)$ or $J_{*}$ [24. With these conditions (conclusions) in place, we can then identify the knot using the A-polynomial and the Alexander polynomial (in particular the genus of the knot). The proof of Theorems 1.1 will be given in Section 3 after we prepare some general properties on $A$-polynomials in Section 2 where we also derive a cabling formula for $A$-polynomials of cabled knots in $S^{3}$ (Theorem 2.10) and in particular we give explicitly the A-polynomials of iterated torus knots (Corollary 2.14). The argument for Theorem 1.2 is more involved than that for Theorem 1.1. for which we need to make some more preparations (besides those made in Section 21) in the next three sections. In Section 4 we collect some topological properties about the family of knots $k(l, m, n, p)$, in particular we give a complete genus formula for $k(l, m, n, p)$ and show that $k\left(l_{*},-1,0,0\right)$ is a class of small knots in $S^{3}$. In Section 5 we collect some info about the $A$-polynomials of the knots $k(l, m, n, p)$ without knowing the explicit formulas of the $A$-polynomials, and with such info we are able to show that if a hyperbolic knot $K$ has the same $A$-polynomial as a given $J_{*}=k\left(l_{*},-1,0,0\right)$, then $K$ has the same half-integral toroidal surgery slope as $J_{*}$ and $K$ is one of $k(l, m, 0, p)$ with $l$ being divisible by $2 p-1$. We then in Section [6 identify each $J_{*}=k\left(l_{*},-1,0,0\right)$ among $k(l, m, 0, p)$ with $(2 p-1) \mid l$ using the genus formula and the half-integral toroidal slope formula for $k(l, m, 0, p)$. Results obtained in these three sections, together with some results from Section 2 are applied in Section 7 to complete the proof of Theorem 1.2 .

Note that the $A$-polynomial $A_{\mathrm{K}}(x, y)$ (for a knot $K$ in $S^{3}$ ) used in this paper is a slightly modified version of the original $A$-polynomial given in 8 . The only difference is that in the current version, the $A$-polynomial of the unknot is 1 and $y-1$ may possibly occur as a factor in $A_{\mathrm{K}}(x, y)$ for certain knots $K$ contributed by some component of the character variety of the knot exterior containing characters of irreducible representations; while in the original version $y-1$ is a factor of the $A$-polynomial for every knot contributed by the unique component of the character variety of the knot exterior consisting of characters of reducible representations (see Section 2 for details). The current version contains a bit more information than the original one.

Acknowledgements. The first author was partially supported by NSF grant numbers DMS-1103976, DMS-1252992, and an Alfred P. Sloan Research Fellowship.

\section{Some properties of $A$-polynomials}

First we need to recall some background material on $A$-polynomials and set up some notations. For a finitely generated group $\Gamma, R(\Gamma)$ denotes the set of representations (i.e. group homomorphisms) from $\Gamma$ to $S L_{2}(\mathbb{C})$. For each representation $\rho \in R(\Gamma)$, its character $\chi_{\rho}$ is the complex valued function $\chi_{\rho}: \Gamma \rightarrow \mathbb{C}$ defined by $\chi_{\rho}(\gamma)=\operatorname{trace}(\rho(\gamma))$ for $\gamma \in \Gamma$. Let $X(\Gamma)$ be the set of characters of representations in $R(\Gamma)$ and $t: R(\Gamma) \rightarrow X(\Gamma)$ the map sending $\rho$ to $\chi_{\rho}$. Then both $R(\Gamma)$ and $X(\Gamma)$ are complex affine algebraic sets such that $t$ is a regular map (see [10] for details).

For an element $\gamma \in \Gamma$, the function $f_{\gamma}: X(\Gamma) \rightarrow \mathbb{C}$ is defined by $f_{\gamma}\left(\chi_{\rho}\right)=\left(\chi_{\rho}(\gamma)\right)^{2}-4$ for each $\chi_{\rho} \in X(\Gamma)$. Each $f_{\gamma}$ is a regular function on $X(\Gamma)$. Obviously $\chi_{\rho} \in X(\Gamma)$ is a zero point of $f_{\gamma}$ if and only if either $\rho(\gamma)= \pm I$ or $\rho(\gamma)$ is a parabolic element. It is also evident that $f_{\gamma}$ is invariant when $\gamma$ is replaced by a conjugate of $\gamma$ or the inverse of $\gamma$.

Note that if $\phi: \Gamma \rightarrow \Gamma^{\prime}$ is a group homomorphism between two finitely generated groups, it naturally induces a regular map $\widetilde{\phi}: R\left(\Gamma^{\prime}\right) \rightarrow R(\Gamma)$ by $\widetilde{\phi}\left(\rho^{\prime}\right)=\rho^{\prime} \circ \phi$ and a regular map $\widehat{\phi}: X\left(\Gamma^{\prime}\right) \rightarrow X(\Gamma)$ by $\widehat{\phi}\left(\chi_{\rho^{\prime}}\right)=\chi_{\widetilde{\phi}\left(\rho^{\prime}\right)}$. Note that if $X_{0}$ is an irreducible subvariety of $X\left(\Gamma^{\prime}\right)$, then the Zariski closure of $\widehat{\phi}\left(X_{0}\right)$ in $X(\Gamma)$ is also irreducible. If in addition the homomorphism $\phi$ is surjective, each of the regular maps $\widetilde{\phi}$ and $\widehat{\phi}$ is an embedding, in which case we may simply consider $R\left(\Gamma^{\prime}\right)$ and $X\left(\Gamma^{\prime}\right)$ as subsets of $R(\Gamma)$ and $X(\Gamma)$ respectively, and write $R\left(\Gamma^{\prime}\right) \subset R(\Gamma)$ and $X\left(\Gamma^{\prime}\right) \subset X(\Gamma)$.

For a compact manifold $W$, we use $R(W)$ and $X(W)$ to denote $R\left(\pi_{1}(W)\right)$ and $X\left(\pi_{1}(W)\right)$ respectively. The $A$-polynomial was introduced in 8. We slightly modify its original definition for a knot $K$ in $S^{3}$ as follows. Let $M_{\mathrm{K}}$ be the exterior of $K$ in $S^{3}$ and let $\{\mu, \lambda\}$ be the standard meridian-longitude 
basis for $\pi_{1}\left(\partial M_{\mathrm{K}}\right)$. Let $\hat{i}_{*}: X\left(M_{\mathrm{K}}\right) \rightarrow X\left(\partial M_{\mathrm{K}}\right)$ be the regular map induced by the inclusion induced homomorphism $i_{*}: \pi_{1}\left(\partial M_{\mathrm{K}}\right) \rightarrow \pi_{1}\left(M_{\mathrm{K}}\right)$, and let $\Lambda$ be the set of diagonal representations of $\pi_{1}\left(\partial M_{\mathrm{K}}\right)$, i.e.

$$
\Lambda=\left\{\rho \in R\left(\partial M_{\mathrm{K}}\right) ; \rho(\mu), \rho(\lambda) \text { are both diagonal matices }\right\} .
$$

Then $\Lambda$ is a subvariety of $R\left(\partial M_{\mathrm{K}}\right)$ and $\left.t\right|_{\Lambda}: \Lambda \rightarrow X\left(\partial M_{\mathrm{K}}\right)$ is a degree 2 , surjective, regular map. We may identify $\Lambda$ with $\mathbb{C}^{*} \times \mathbb{C}^{*}$ through the eigenvalue map $E: \Lambda \rightarrow \mathbb{C}^{*} \times \mathbb{C}^{*}$ which sends $\rho \in \Lambda$ to $(x, y) \in \mathbb{C}^{*} \times \mathbb{C}^{*}$ if $\rho(\mu)=\left(\begin{array}{cc}x & 0 \\ 0 & x^{-1}\end{array}\right)$ and $\rho(\lambda)=\left(\begin{array}{cc}y & 0 \\ 0 & y^{-1}\end{array}\right)$. For every knot in $S^{3}$, there is a unique component in $X\left(M_{\mathrm{K}}\right)$ consisting of characters of reducible representations, which we call the trivial component of $X\left(M_{\mathrm{K}}\right)$ (The trivial component is of dimensional one). Now let $X^{*}\left(M_{\mathrm{K}}\right)$ be the set of nontrivial components of $X\left(M_{\mathrm{K}}\right)$ each of which has a 1-dimensional image in $X\left(\partial M_{\mathrm{K}}\right)$ under the map $\hat{i}_{*}$. The set $X^{*}\left(M_{\mathrm{K}}\right)$ is possibly empty, and in fact with the current knowledge it is known that $X^{*}\left(M_{\mathrm{K}}\right)$ is empty if and only if $K$ is the unknot. So when $K$ is a nontrivial knot, $\left(\left.t\right|_{\Lambda}\right)^{-1}\left(\widehat{i}_{*}\left(X^{*}\left(M_{\mathrm{K}}\right)\right)\right.$ is 1-dimensional in $\Lambda$ and in turn $E\left(\left(\left.t\right|_{\Lambda}\right)^{-1}\left(\widehat{i}_{*}\left(X^{*}\left(M_{\mathrm{K}}\right)\right)\right)\right.$ is 1-dimensional in $\mathbb{C}^{*} \times \mathbb{C}^{*} \subset \mathbb{C} \times \mathbb{C}$. Let $D$ be the Zariski closure of $E\left(\left(\left.t\right|_{\Lambda}\right)^{-1}\left(\hat{i}_{*}\left(X^{*}\left(M_{\mathrm{K}}\right)\right)\right)\right.$ in $\mathbb{C}^{2}$. Then $D$ is a plane curve in $\mathbb{C}^{2}$ defined over $\mathbb{Q}$. Let $A_{\mathrm{K}}(x, y)$ be the defining polynomial of $D$ normalized so that $A_{\mathrm{K}}(x, y) \in \mathbb{Z}[x, y]$ with no repeated factors and with 1 as the greatest common divisor of its coefficients. Then $A_{\mathrm{K}}(x, y)$ is uniquely defined up to sign. For the unknot we define its $A$-polynomial to be 1 . As remarked in the introduction section, $y-1$ might occur as a factor of $A_{\mathrm{K}}(x, y)$ for certain knots. Also by [3] [11, $A_{\mathrm{K}}(x, y)=1$ if and only if $K$ is the unknot (in fact for every nontrivial knot $K, A_{\mathrm{K}}(x, y)$ contains a nontrivial factor which is not $\left.y-1\right)$.

We note that from the constructional definition of the $A$-polynomial we see that each component $X_{0}$ of $X^{*}\left(M_{\mathrm{K}}\right)$ contributes a factor $f_{0}(x, y)$ in $A_{\mathrm{K}}(x, y)$, i.e. $f_{0}(x, y)$ is the defining polynomial of the plane curve $D_{0}$ which is the Zariski closure of $E\left(\left(\left.t\right|_{\Lambda}\right)^{-1}\left(\widehat{i}_{*}\left(X_{0}\right)\right)\right)$ in $\mathbb{C}^{2}$, and moreover $f_{0}(x, y)$ is balanced, i.e. if $(x, y)$ is a generic zero point of $f_{0}(x, y)$ then $\left(x^{-1}, y^{-1}\right)$ is also a zero point of $f_{0}(x, y)$. Also note that $f_{0}(x, y)$ is not necessarily irreducible over $\mathbb{C}$ but contains at most two irreducible factors over $\mathbb{C}$. We shall call such $f_{0}(x, y)$ a balanced-irreducible factor of $A_{\mathrm{K}}(x, y)$. Obviously $A_{\mathrm{K}}(x, y)$ is a product of balanced-irreducible factors and the product decomposition is unique up to the ordering of the factors.

We now define a couple of functions which will be convenient to use in expressing the $A$-polynomials for torus knots and later on for cabled knots and iterated torus knots. Let $(p, q)$ be a pair of relative prime integers with $q \geq 2$. Define $F_{(p, q)}(x, y) \in \mathbb{Z}[x, y]$ to be the polynomial determined by the pair $(p, q)$ as follows:

$$
F_{(p, q)}(x, y)= \begin{cases}1+x^{2 p} y, & \text { if } q=2, p>0 \\ x^{-2 p}+y, & \text { if } q=2, p<0 \\ -1+x^{2 p q} y^{2}, & \text { if } q>2, p>0 \\ -x^{-2 p q}+y^{2}, & \text { if } q>2, p<0\end{cases}
$$

and define $G_{(p, q)}(x, y) \in \mathbb{Z}[x, y]$ to be the polynomial determined by the pair $(p, q)$ as follows:

$$
G_{(p, q)}(x, y)= \begin{cases}-1+x^{p q} y, & \text { if } p>0 \\ -x^{-p q}+y, & \text { if } p<0 .\end{cases}
$$

Note that the ring $\mathbb{C}[x, y]$ is a unique factorization domain. The following lemma can be easily checked.

Lemma 2.1. Among the polynomials in (1) and (2), the first two in (1) and the two in (2) are irreducible over $\mathbb{C}$, and the last two in (1) can be factorized as the product of two irreducible polynomials over $\mathbb{C}$ :

$$
\begin{aligned}
& -1+x^{2 p q} y^{2}=\left(-1+x^{p q} y\right)\left(1+x^{p q} y\right), \quad \text { if } q>2, p>0 \text {, } \\
& -x^{-2 p q}+y^{2}=\left(-x^{-p q}+y\right)\left(x^{-p q}+y\right), \quad \text { if } q>2, p<0 \text {. }
\end{aligned}
$$

The set of nontrivial torus knots $T(p, q)$ are naturally indexed by pairs $(p, q)$ satisfying $|p|>q \geq 2$, $(p, q)=1$. Note that $T(-p, q)$ is the mirror image of $T(p, q)$. The $A$-polynomial of a torus knot $T(p, q)$ is given by (e.g. [28, Example 4.1]):

$$
A_{T(p, q)}(x, y)=F_{(p, q)}(x, y)
$$


In particular the $A$-polynomial distinguishes $T(p, q)$ from $T(-p, q)$.

For the exterior $M_{\mathrm{K}}$ of a nontrivial knot in $S^{3}, H_{1}\left(\partial M_{\mathrm{K}} ; \mathbb{Z}\right) \cong \pi_{1}\left(\partial M_{\mathrm{K}}\right)$ can be considered as a subgroup of $\pi_{1}\left(M_{\mathrm{K}}\right)$ which is well defined up to conjugation. In particular, the function $f_{\alpha}$ on $X\left(M_{\mathrm{K}}\right)$ is well defined for each class $\alpha \in H_{1}\left(\partial M_{\mathrm{K}} ; \mathbb{Z}\right)$. As $f_{\alpha}$ is also invariant under the change of the orientation of $\alpha, f_{\alpha}$ is also well defined when $\alpha$ is a slope in $\partial M_{\mathrm{K}}$. Later on for convenience we will often not make a distinction among a primitive class of $H_{1}\left(\partial M_{\mathrm{K}} ; \mathbb{Z}\right)$, the corresponding element of $\pi_{1}\left(\partial M_{\mathrm{K}}\right)$ and the corresponding slope in $\partial M_{\mathrm{K}}$, so long as it is well defined.

It is known (e.g. [6]) that any irreducible curve $X_{0}$ in $X\left(M_{\mathrm{K}}\right)$ belongs to one of the following three mutually exclusive types:

(a) for each slope $\alpha$ in $\partial M_{\mathrm{K}}$, the function $f_{\alpha}$ is non-constant on $X_{0}$;

(b) there is a unique slope $\alpha_{0}$ in $\partial M_{\mathrm{K}}$ such that the function $f_{\alpha_{0}}$ is constant on $X_{0}$;

(c) for each slope $\alpha$ in $\partial M_{\mathrm{K}}$, the function $f_{\alpha}$ is constant on $X_{0}$.

Obviously a curve of type (a) or (b) has one dimensional image in $X\left(\partial M_{\mathrm{K}}\right)$ under the map $\widehat{i}_{*}$. Note that the trivial component of $X\left(M_{\mathrm{K}}\right)$ is of type (b). Hence a curve of type (a) is contained in $X^{*}\left(M_{\mathrm{K}}\right)$ and so is a curve of type (b) if it is not the trivial component of $X\left(M_{\mathrm{K}}\right)$.

An irreducible curve in case (a) is named a norm curve. Indeed as the name indicates, a norm curve in $X\left(M_{\mathrm{K}}\right)$ can be used to define a norm, known as Culler-Shalen norm, on the real 2-dimensional plane $H_{1}\left(\partial M_{\mathrm{K}} ; \mathbb{R}\right)$ satisfying certain properties. Such curve exists when $M_{\mathrm{K}}$ is hyperbolic, namely any component of $X\left(M_{\mathrm{K}}\right)$ which contains the character of a discrete faithful representation of $\pi_{1}\left(M_{\mathrm{K}}\right)$ is a norm curve.

For an irreducible curve $X_{0}$ in $X\left(M_{\mathrm{K}}\right)$, let $\tilde{X}_{0}$ be the smooth projective completion of $X_{0}$ and let $\phi: \tilde{X}_{0} \rightarrow X_{0}$ be the birational isomorphism. The map $\phi$ is onto and is defined at all but finitely many points of $\tilde{X}_{0}$. The points of $\tilde{X}_{0}$ where $\phi$ is not defined are called ideal points and all other points of $\tilde{X}_{0}$ are called regular points. The map $\phi$ induces an isomorphism from the function field of $X_{0}$ to that of $\tilde{X}_{0}$. In particular every regular function $f_{\gamma}$ on $X_{0}$ corresponds uniquely to its extension $\tilde{f}_{\gamma}$ on $\tilde{X}_{0}$ which is a rational function. If $\tilde{f}_{\gamma}$ is not a constant function on $\tilde{X}_{0}$, its degree, denoted $\operatorname{deg}\left(\tilde{f}_{\gamma}\right)$, is equal to the number of zeros of $\tilde{f}_{\gamma}$ in $\tilde{X}_{0}$ counted with multiplicity, i.e.

$$
\operatorname{deg}\left(\tilde{f}_{\gamma}\right)=\sum_{v \in \tilde{X}_{0}} Z_{v}\left(\tilde{f}_{\gamma}\right)
$$

where $Z_{v}\left(\tilde{f}_{\gamma}\right)$ is the zero degree of $\tilde{f}_{\gamma}$ at point $v \in \tilde{X}_{0}$.

We shall identify $H_{1}\left(\partial M_{\mathrm{K}} ; \mathbb{R}\right)$ with the real $x y$-plane so that $H_{1}\left(\partial M_{\mathrm{K}} ; \mathbb{Z}\right)$ are integer lattice points with $\mu=(1,0)$ being the meridian class and $\lambda=(0,1)$ the longitude class. So each slope $m / n$ corresponds to the pair of primitive elements $\pm(m, n) \in H_{1}\left(\partial M_{\mathrm{K}} ; \mathbb{Z}\right)$.

Theorem 2.2. Let $X_{0}$ be a norm curve of $X\left(M_{K}\right)$. Then the associated Culler-Shalen norm $\|\cdot\|_{0}$ on $H_{1}\left(\partial M_{K} ; \mathbb{R}\right)$ has the following properties: let

$$
s_{0}=\min \left\{\|\alpha\|_{0} ; \alpha \neq 0, \alpha \in H_{1}\left(\partial M_{K} ; \mathbb{Z}\right)\right\}
$$

and let $B_{0}$ be the disk in $H_{1}\left(\partial M_{K} ; \mathbb{R}\right)$ with radius $s_{0}$ with respect to the norm $\|\cdot\|_{0}$, then

(1) For each nontrivial element $\alpha=(m, n) \in H_{1}\left(\partial M_{K} ; \mathbb{Z}\right),\|\alpha\|_{0}=\operatorname{deg}\left(\tilde{f}_{\alpha}\right) \neq 0$ and thus $\|\alpha\|_{0}=\|-\alpha\|_{0}$.

(2) The disk $B_{0}$ is a convex finite sided polygon symmetric to the origin whose interior does not contain any non-zero element of $H_{1}\left(\partial M_{K} ; \mathbb{Z}\right)$ and whose boundary contains at least one but at most four nonzero classes of $H_{1}\left(\partial M_{K} ; \mathbb{Z}\right)$ up to sign.

(3) If $(a, b)$ is a vertex of $B_{0}$, then there is a boundary slope $m / n$ of $\partial M_{K}$ such that $\pm(m, n)$ lie in the line passing through $(a, b)$ and $(0,0)$. (That is, $a / b$ is a boundary slope of $\partial M_{K}$ for any vertex $(a, b)$ of $\left.B_{0}\right)$.

(4) If a primitive class $\alpha=(m, n) \in H_{1}\left(\partial M_{K} ; \mathbb{Z}\right)$ is not a boundary class and $M_{K}(\alpha)$ has no noncyclic representations, then $\alpha=(m, n)$ lies in $\partial B$ (i.e. $\left.\|\alpha\|_{0}=s_{0}\right)$ and is not a vertex of $B_{0}$.

(5) If the meridian class $\mu=(1,0)$ is not a boundary class, then for any non-integral class $\alpha=(m, n)$ if it is not a vertex of $B_{0}$ then it does not lie in $\partial B$ and thus $\|\alpha\|_{0}>\|\mu\|_{0}=s_{0}$. 
Theorem 2.2 is originated from [9. Chatper 1] although it was assumed there that the curve $X_{0}$ contains the character of a discrete faithful representation of $M_{\mathrm{K}}$. The version given here is contained in [6].

Recall that if $f_{0}(x, y)=\sum a_{i, j} x^{i} y^{j} \in \mathbb{C}[x, y]$ is a two variable polynomial in $x$ and $y$ with complex coefficients, the Newton polygon $N_{0}$ of $f_{0}(x, y)$ is defined to be the convex hull in the real $x y$-plane of the set of points

$$
\left\{(i, j) ; a_{i, j} \neq 0\right\}
$$

The following theorem is proved in [4].

Theorem 2.3. Let $X_{0}$ be a norm curve of $X\left(M_{K}\right)$ and let $f_{0}(x, y)$ be the balanced-irreducible factor of $A_{K}(x, y)$ contributed by $X_{0}$. Then the norm polygon $B_{0}$ determined by $X_{0}$ is dual to the Newton polygon $N_{0}$ of $f_{0}(x, y)$ in the following way: the set of slopes of vertices of $B_{0}$ is equal to the set of slopes of edges of $N_{0}$. In fact $B_{0}$ and $N_{0}$ mutually determine each other up a positive integer multiple.

We remark that although in 4 there was some additional condition imposed on $X_{0}$ and the version of the $A$-polynomial defined in [4] is mildly different from the one as given here, the above theorem remains valid with identical reasoning as given in 4 . We only need to describe the exact relation between $B_{0}$ and $N_{0}$ as follows, to see how they determine each other up to an integer multiple. Let $Y_{0}$ be the Zariski closure of the restriction $\widehat{i}_{*}\left(X_{0}\right)$ of $X_{0}$ in $X\left(\partial M_{\mathrm{K}}\right)$ and let $d_{0}$ be the degree of the map $\widehat{i}_{*}: X_{0} \rightarrow Y_{0}$. As explained in 4 . (originated from [26]), the Newton polygon $N_{0}$ determines a width function $w$ on the set of slopes given by

$$
w(p / q)=k \in \mathbb{Z}
$$

if $k+1$ is the number of lines in the $x y$-plane of the slope $q / p$ which contain points of both $\mathbb{Z}^{2}$ and $N_{0}$. The width function in turn defines a norm $\|\cdot\|_{N_{0}}$ on the $x y$-plane $H_{1}\left(\partial M_{\mathrm{K}} ; \mathbb{R}\right)$ such that

$$
\|(p, q)\|_{N_{0}}=w(p / q)
$$

for each primitive class $(p, q) \in H_{1}\left(\partial M_{\mathrm{K}} ; \mathbb{Z}\right)$. Finally

$$
\|\cdot\|_{0}=2 d_{0}\|\cdot\|_{N_{0}} .
$$

Corollary 2.4. If every balanced-irreducible factor of $A_{K}(x, y)$ over $\mathbb{C}$ has two monomials, then $K$ is not a hyperbolic knot.

Proof. The condition of the corollary means that the Newton polygon of each balanced-irreducible factor of $A_{K}(x, y)$ consists of a single edge. On the other hand for a hyperbolic knot, its character variety contains a norm curve component $X_{0}$ which contributes a balanced-irreducible factor $f_{0}(x, y)$ to the $A$-polynomial such that the Newton polygon of $f_{0}(x, y)$ has at least two edges of different slopes. $\diamond$

An irreducible curve in $X^{*}\left(M_{\mathrm{K}}\right)$ of type (b) (such curve exists only for certain knots) is named a seminorm curve as suggested by the following theorem which is contained in [6].

Theorem 2.5. Suppose that $X_{0} \subset X^{*}\left(M_{K}\right)$ is an irreducible curve of type (b) with $\alpha_{0}$ being the unique slope such that $f_{\alpha_{0}}$ is constant on $X_{0}$. Then a semi-norm $\|\cdot\|_{0}$ can be defined on $H_{1}\left(\partial M_{K} ; \mathbb{R}\right)$, with the following properties:

(1) For each slope $\alpha \neq \alpha_{0},\|\alpha\|_{0}=\operatorname{deg}\left(\tilde{f}_{\alpha}\right) \neq 0$.

(2) For the unique slope $\alpha_{0}$ associated to $X_{0},\left\|\alpha_{0}\right\|_{0}=0$ and $\alpha_{0}$ is a boundary slope of $M_{K}$.

(3) If $\alpha$ is a primitive class and is not a boundary class and $M_{K}(\alpha)$ has no non-cyclic representation, then $\Delta\left(\alpha, \alpha_{0}\right)=1$.

(4) Let $s_{0}=\min \left\{\|\alpha\|_{0} ; \alpha \neq \alpha_{0}\right.$ is a slope $\}$. Then for any slope $\alpha,\|\alpha\|_{0}=s_{0} \Delta\left(\alpha, \alpha_{0}\right)$.

Note that for each torus knot $T(p, q)$, every nontrivial component in its character variety is a semi-norm curve with $p q$ as the associated slope.

Remark 2.6. If $K$ is a small knot, i.e. if its exterior $M_{\mathrm{K}}$ does not contain any closed essential surface, then every nontrivial component of $X\left(M_{\mathrm{K}}\right)$ is either a norm curve or a semi-norm curve. 
We now proceed to get some properties on $A$-polynomials of satellite knots in $S^{3}$. Recall that a knot $K$ in $S^{3}$ is a satellite knot if there is a pair of knots $C$ and $P$ in $S^{3}$, called a companion knot and a pattern knot respectively, associated to $K$, such that $C$ is nontrivial, $P$ is contained in a trivial solid torus $V$ in $S^{3}$ but is not contained in a 3-ball of $V$ and is not isotopic to the core circle of $V$, and there is a homeomorphism $f$ from $V$ to a regular neighborhood $N(C)$ of $C$ in $S^{3}$ which maps a longitude of $V$ (which bounds a disk in $S^{3}$ ) to a longitude of $N(C)$ (which bounds a Seifert surface for $C$ ) and maps a meridian of $V$ to a meridian of $N(C)$, and finally $K=f(P)$. We sometimes write a satellite knot as $K=(P, C, V, f)$ to include the above defining information ( $K$ still depends on how $P$ is embedded in $V)$.

Lemma 2.7. Let $K=(P, C, V, f)$ be a satellite knot in $S^{3}$. Then $A_{P}(x, y) \mid A_{K}(x, y)$ in $\mathbb{Z}[x, y]$.

Proof. The lemma is obviously true when $P$ is the unknot in $S^{3}$. So we may assume that $P$ is a nontrivial knot in $S^{3}$. Let $M_{\mathrm{K}}, M_{\mathrm{C}}, M_{\mathrm{P}}$ be the exteriors of $K, C$ and $P$ in $S^{3}$ respectively. There is a degree one map $h:\left(M_{\mathrm{K}}, \partial M_{\mathrm{K}}\right) \rightarrow\left(M_{\mathrm{P}}, \partial M_{\mathrm{P}}\right)$ such that $h^{-1}\left(\partial M_{\mathrm{P}}\right)=\partial M_{\mathrm{K}}$ and $h \mid: \partial M_{\mathrm{K}} \rightarrow \partial M_{\mathrm{P}}$ is a homeomorphism. The map $h$ is given by a standard construction as follows. From the definition of the pattern knot given above, we see that if $W$ is the exterior of $P$ in the trivial solid torus $V$, then $M_{\mathrm{P}}$ is obtained by Dehn filling $W$ along $\partial V$ with a solid torus $V^{\prime}$ such that the meridian slope of $V^{\prime}$ is identified with the longitude slope of $V$. Also if we let $Y=f(W)$, then $M_{\mathrm{K}}=M_{\mathrm{C}} \cup Y$. Now the degree one map $h: M_{\mathrm{K}} \rightarrow M_{\mathrm{P}}$ is defined to be: on $Y$ it is the homeomorphism $f^{-1}: Y \rightarrow W$ and on $M_{\mathrm{C}}$ it maps a regular neighborhood of a Seifert surface in $M_{\mathrm{C}}$ to a regular neighborhood of a meridian disk of $V^{\prime}$ in $V^{\prime}$ and maps the rest of $M_{\mathrm{C}}$ onto the rest of $V^{\prime}$ (which is a 3-ball).

The degree one map $h$ induces a surjective homomorphism $h_{*}$ from $\pi_{1}\left(M_{\mathrm{K}}\right)$ to $\pi_{1}\left(M_{\mathrm{P}}\right)$ such that

$$
h_{*} \mid: \pi_{1}\left(\partial M_{\mathrm{K}}\right) \rightarrow \pi_{1}\left(\partial M_{\mathrm{P}}\right)
$$

is an isomorphism, mapping the meridian to the meridian and the longitude to the longitude. In turn $h_{*}$ induces an embedding $\widehat{h_{*}}$ of $X\left(M_{\mathrm{P}}\right)$ into $X\left(M_{\mathrm{K}}\right)$ in such a way that the restriction of $\widehat{h_{*}}\left(X\left(M_{\mathrm{P}}\right)\right)$ on $X\left(\partial M_{\mathrm{K}}\right)$ with respect to the standard meridian-longitude basis $\left\{\mu_{\mathrm{K}}, \lambda_{\mathrm{K}}\right)$ of $\partial M_{\mathrm{K}}$ is the same as the restriction of $X\left(M_{\mathrm{P}}\right)$ on $X\left(\partial M_{\mathrm{P}}\right)$ with respect to the standard meridian-longitude basis $\left\{\mu_{\mathrm{P}}, \lambda_{\mathrm{P}}\right\}$ of $\partial M_{\mathrm{P}}$, i.e. for each $\chi_{\rho} \in X\left(M_{\mathrm{P}}\right)$, we have $\chi_{\rho}\left(\mu_{\mathrm{P}}\right)=\widehat{h_{*}}\left(\chi_{\rho}\right)\left(\mu_{\mathrm{K}}\right), \chi_{\rho}\left(\lambda_{\mathrm{P}}\right)=\widehat{h_{*}}\left(\chi_{\rho}\right)\left(\lambda_{\mathrm{K}}\right)$ and $\chi_{\rho}\left(\mu_{\mathrm{P}} \lambda_{\mathrm{P}}\right)=$ $\widehat{h_{*}}\left(\chi_{\rho}\right)\left(\mu_{\mathrm{K}} \lambda_{\mathrm{K}}\right)$. The conclusion of the lemma now follows easily from the constructional definition of the $A$-polynomial. $\diamond$

For polynomials $f(x, \bar{y}) \in \mathbb{C}[x, \bar{y}]$ and $g(y, \bar{y}) \in \mathbb{C}[y, \bar{y}]$ both with nonzero degree in $\bar{y}$, let

$$
\operatorname{Res}_{\bar{y}}(f(x, \bar{y}), g(y, \bar{y}))
$$

denote the resultant of $f(x, \bar{y})$ and $g(y, \bar{y})$ eliminating the variable $\bar{y}$. In general $\operatorname{Res} \bar{y}(f(x, \bar{y}), g(y, \bar{y}))$ may have repeated factors even when both $f(x, \bar{y})$ and $g(y, \bar{y})$ are irreducible over $\mathbb{C}$. For a polynomial $f(x, y) \in \mathbb{C}[x, y]$, let

$$
\operatorname{Red}[f(x, y)]
$$

denote the polynomial obtained from $f(x, y)$ by deleting all its repeated factors.

Proposition 2.8. Let $K=(P, C, V, f)$ be a satellite knot such that the winding number $w$ of $P$ in the solid torus $V$ is non-zero. Then every balanced-irreducible factor $f_{C}(\bar{x}, \bar{y})$ of the A-polynomial $A_{C}(\bar{x}, \bar{y})$ of $C$ extends to a balanced factor $f_{K}(x, y)$ of the A-polynomial $A_{K}(x, y)$ of $K$. More precisely,

(1) if the $\bar{y}$-degree of $f_{C}(\bar{x}, \bar{y})$ is non-zero, then $f_{K}(x, y)=\operatorname{Red}\left[\operatorname{Res} \bar{y}\left(f_{C}\left(x^{w}, \bar{y}\right), \bar{y}^{w}-y\right)\right]$. In particular if $f_{C}(\bar{x}, \bar{y})=\bar{y}+\delta \bar{x}^{n}$ or $\bar{y} \bar{x}^{n}+\delta$ for some nonnegative integer $n$ and $\delta \in\{1,-1\}$ (such factor is irreducible and balanced), then $f_{K}(x, y)=y-(-\delta)^{w} x^{n w^{2}}$ or $y x^{n w^{2}}-(-\delta)^{w}$ respectively;

(2) if the $\bar{y}$-degree of $f_{C}(\bar{x}, \bar{y})$ is zero, i.e. $f_{C}(\bar{x}, \bar{y})=f_{C}(\bar{x})$ is a function of $\bar{x}$ only, then $f_{K}=f_{C}\left(x^{w}\right)$.

Proof. Let $M_{\mathrm{K}}, M_{\mathrm{C}}, Y, \mu_{\mathrm{C}}, \lambda_{\mathrm{C}}, \mu_{\mathrm{K}}, \lambda_{\mathrm{K}}$ be defined as in the proof of Lemma 2.7 We have $M_{\mathrm{K}}=M_{\mathrm{C}} \cup Y$. Note that $H_{1}(Y ; \mathbb{Z})=\mathbb{Z}\left[\mu_{\mathrm{K}}\right] \oplus \mathbb{Z}\left[\lambda_{\mathrm{C}}\right]$, and $\left[\lambda_{\mathrm{K}}\right]=w\left[\lambda_{\mathrm{C}}\right],\left[\mu_{\mathrm{C}}\right]=w\left[\mu_{\mathrm{K}}\right]$. Given a balanced-irreducible factor $f_{\mathrm{C}}(\bar{x}, \bar{y})$ of $A_{\mathrm{C}}(\bar{x}, \bar{y})$, let $X_{0}$ be a component of $X^{*}\left(M_{\mathrm{C}}\right)$ which gives rise the factor $f_{\mathrm{C}}(\bar{x}, \bar{y})$. For each 
element $\chi_{\rho} \in X_{0}$, the restriction of $\rho$ on $\pi_{1}\left(\partial M_{\mathrm{C}}\right)$ can be extended to an abelian representation of $\pi_{1}(Y)$, and thus $\rho$ can be extended to a representation of $\pi_{1}\left(M_{\mathrm{K}}\right)$, which we still denote by $\rho$, such that

$$
\rho\left(\mu_{\mathrm{C}}\right)=\rho\left(\mu_{\mathrm{K}}^{w}\right), \rho\left(\lambda_{\mathrm{K}}\right)=\rho\left(\lambda_{\mathrm{C}}^{w}\right) .
$$

It follows that $X_{0}$ extends to a component or components of $X^{*}\left(M_{\mathrm{K}}\right)$ whose restriction on $\partial M_{\mathrm{K}}$ is or are one dimensional and thus all together gives rise a balanced factor $f_{\mathrm{K}}(x, y)$ of $A_{\mathrm{K}}(x, y)$. Moreover the variables $(x, y)$ of $f_{\mathrm{K}}(x, y)$ and the variables $\bar{x}$ and $\bar{y}$ of $f_{\mathrm{C}}(\bar{x}, \bar{y})$ are related by

$$
\bar{x}=x^{w}, y=\bar{y}^{w} .
$$

Therefore when the $\bar{y}$-degree of $f_{\mathrm{C}}(\bar{x}, \bar{y})$ is positive, $f_{\mathrm{K}}(x, y)$ can be obtained by taking the resultant of $f_{\mathrm{C}}\left(x^{w}, \bar{y}\right)$ and $\bar{y}^{w}-y$, eliminating the variable $\bar{y}$, and then deleting possible repeated factors. In particular if $f_{\mathrm{C}}(\bar{x}, \bar{y})=\bar{y}+\delta \bar{x}^{n}$ or $\bar{y} \bar{x}^{n}+\delta$ for some nonnegative integer $n$ and $\delta \in\{-1,1\}$, then the resultant of $\bar{y}+\delta x^{w n}$ or $\bar{y} x^{w n}+\delta$ with $\bar{y}^{w}-y$, eliminating the variable $\bar{y}$, is $y-(-\delta)^{w} x^{n w^{2}}$ or $y x^{n w^{2}}-(-\delta)^{w}$ respectively (which is irreducible over $\mathbb{C}$ and is banlanced). Also if the degree of $f_{\mathrm{C}}$ in $\bar{y}$ is zero, then obviously $f_{\mathrm{K}}=f_{\mathrm{C}}\left(x^{w}\right)$.

Remark 2.9. In Proposition 2.8 the $y$-degree of $f_{\mathrm{K}}(x, y)$ is at most equal to the $\bar{y}$-degree of $f_{\mathrm{C}}(\bar{x}, \bar{y})$ (and generically they are equal). This follows directly from the definition of the resultant (cf. [22, $\mathrm{IV}, \S 8])$.

Next we are going to consider cabled knots. Let $(p, q)$ be a pair of relatively prime integers with $|q| \geq 2$, and $K$ be the $(p, q)$-cabled knot over a nontrivial knot $C$. That is, $K$ is a satellite knot with $C$ as a companion knot and with $T(p, q)$ as a pattern knot which lies in the defining solid torus $V$ as a standard $(p, q)$-cable with winding number $|q|$. As the $(-p,-q)$-cable over a knot is equal to the $(p, q)$-cable over the same knot, we may always assume $q \geq 2$. The following theorem gives a cabling formula for the $A$-polynomial of a cabled knot $K$ over a nontrivial knot $C$, in terms of the $A$-polynomial $A_{\mathrm{C}}(\bar{x}, \bar{y})$ of $C$.

Theorem 2.10. Let $K$ be the $(p, q)$-cabled knot over a nontrivial knot $C$, with $q \geq 2$. Then

$$
A_{K}(x, y)=\operatorname{Red}\left[F_{(p, q)}(x, y) \operatorname{Res} \bar{y}\left(A_{C}\left(x^{q}, \bar{y}\right), \bar{y}^{q}-y\right)\right]
$$

if the $\bar{y}$-degree of $A_{C}(\bar{x}, \bar{y})$ is nonzero and

$$
A_{K}(x, y)=F_{(p, q)}(x, y) A_{C}\left(x^{q}\right)
$$

if the $\bar{y}$-degree of $A_{C}(\bar{x}, \bar{y})$ is zero.

Proof. For a polynomial $f(\bar{x}, \bar{y}) \in \mathbb{C}[\bar{x}, \bar{y}]$, define

$$
\operatorname{Ext}^{q}[f(\bar{x}, \bar{y})]= \begin{cases}\operatorname{Red}\left[\operatorname{Res}_{\bar{y}}\left(f\left(x^{q}, \bar{y}\right), \bar{y}^{q}-y\right)\right], & \text { if the degree of } f(\bar{x}, \bar{y}) \text { in } \bar{y} \text { is nonzero } \\ f\left(x^{q}\right), & \text { if the degree of } f(\bar{x}, \bar{y}) \text { in } \bar{y} \text { is zero }\end{cases}
$$

Then Proposition 2.8 was saying that

$$
f_{\mathrm{K}}(x, y)=E x t^{w}\left[f_{\mathrm{C}}(\bar{x}, \bar{y})\right]
$$

and Theorem 2.10 is saying that

$$
A_{\mathrm{K}}(x, y)=\operatorname{Red}\left[F_{(p, q)}(x, y) \operatorname{Ext}^{q}\left[A_{\mathrm{C}}(\bar{x}, \bar{y})\right]\right] .
$$

Note that $\operatorname{Ext}^{q}\left[A_{\mathrm{C}}(\bar{x}, \bar{y})\right]=\operatorname{Red}\left[\Pi \operatorname{Ext} t^{q}\left[f_{\mathrm{C}}(\bar{x}, \bar{y})\right]\right]$ where the product runs over all balanced-irreducible factors $f_{\mathrm{C}}(\bar{x}, \bar{y})$ of $A_{\mathrm{C}}(\bar{x}, \bar{y})$ and thus by Proposition 2.8, $\operatorname{Ext}^{q}\left[A_{\mathrm{C}}(\bar{x}, \bar{y})\right]$ is a balanced factor of $A_{\mathrm{K}}(x, y)$. So we only need to show

Claim 2.11. $F_{(p, q)}(x, y)$ is a balanced factor of $A_{K}(x, y)$. (Note that each irreducible factor of $F_{(p, q)}(x, y)$ is balanced). 
Claim 2.12. Besides $F_{(p, q)}(x, y)$ and $\operatorname{Ext}^{q}\left[A_{C}(\bar{x}, \bar{y})\right], A_{K}(x, y)$ has no other balanced factors.

To prove the above two claims, let $M_{\mathrm{K}}, M_{\mathrm{C}}, Y, \mu_{\mathrm{C}}, \lambda_{\mathrm{C}}, \mu_{\mathrm{K}}, \lambda_{\mathrm{K}}$ be defined as in Lemma 2.7 with respect to $K=(P, C, f, V)$ where $P=T(p, q)$ is embedded in $V$ as a standard $(p, q)$-cable. We have $M_{\mathrm{K}}=M_{\mathrm{C}} \cup Y$ and $\partial Y=\partial M_{\mathrm{C}} \cup \partial M_{\mathrm{K}}$. For a convenience to the present argument, we give a direct description of $Y$ as follows. We may consider $N=N(C)$ as $D \times C$, where $D$ is a disk of radius 2 , such that $\{x\} \times C$ has slope zero for each point $x \in \partial D$. Let $D_{*}$ be the concentric sub-disk in $D$ with radius 1 . Then $N_{*}=D_{*} \times C$ is a solid torus in $N(C)$ sharing the same core circle $C$. We may assume that the knot $K$ is embedded in the boundary of $N_{*}$ as a standard $(p, q)$-curve, where $\partial N_{*}$ has the meridian-longitude coordinates consistent with that of $\partial M_{\mathrm{C}}=\partial N$ (i.e. for a point $x \in \partial D_{*},\{x\} \times C$ is a longitude of $\partial N_{*}$ ). Then $Y$ is the exterior of $K$ in $N$.

Note that $Y$ is a Seifert fibred space whose base orbifold is an annulus with a single cone point of order $q$, a Seifert fiber of $Y$ in $\partial M_{\mathrm{C}}$ has slope $p / q$ and a Seifert fiber of $Y$ in $\partial M_{\mathrm{K}}$ has slope $p q$. Let $\gamma_{\mathrm{C}}$ be a Seifert fibre of $Y$ lying in $\partial M_{\mathrm{C}}$ and $\gamma_{\mathrm{K}}$ be a Seifert fibre of $Y$ lying in $\partial M_{\mathrm{K}}$. Up to conjugation, we may consider $\gamma_{\mathrm{C}}$ and $\gamma_{\mathrm{K}}$ as elements of $\pi_{1}(Y)$. Also note that $\gamma_{\mathrm{C}}$ is conjugate to $\gamma_{\mathrm{K}}$ in $\pi_{1}(Y)$. It is well known that each of $\gamma_{\mathrm{C}}$ and $\gamma_{\mathrm{K}}$ lies in the center of $\pi_{1}(Y)$ which is independent of conjugation. It follows that if $\rho \in R(Y)$ is an irreducible representation then $\rho\left(\gamma_{\mathrm{C}}\right)=\rho\left(\gamma_{\mathrm{K}}\right)=\epsilon I$, for some fixed $\epsilon \in\{1,-1\}$, where $I$ is the identity matrix. Hence if $X_{0}$ is an irreducible subvariety of $X(Y)$ which contains the character of an irreducible representation, then for every $\chi_{\rho} \in X_{0}$, we have $\rho\left(\gamma_{\mathrm{C}}\right)=\rho\left(\gamma_{\mathrm{K}}\right)=\epsilon I$, which is due to the fact that the characters of irreducible representations are dense in $X_{0}$.

Now we are ready to prove Claim 2.11 If $|p|>1$, then by Lemma 2.7 and the formula (3), $F_{(p, q)}(x, y)$ is a factor of $A_{\mathrm{K}}(x, y)$. So we may assume that $|p|=1$. Under this assumption, one can see that the fundamental group of $Y$ has the following presentation:

$$
\pi_{1}(Y)=<\alpha, \beta \mid \alpha^{q} \beta=\beta \alpha^{q}>
$$

such that

$$
\mu_{\mathrm{K}}=\alpha \beta
$$

where $\alpha$ is a based simple loop free homotopic in $Y$ to the center circle of $N$ and $\beta$ is a based simple loop free homotopic in $Y$ to $\lambda_{\mathrm{C}}$ in $\partial M_{\mathrm{C}}$. To see these assertions, note that $Y$ contains the essential annulus $A_{2}=\partial N_{*} \cap Y$ with $\partial A_{2} \subset \partial M_{\mathrm{K}}$ of the slope $p q$ (the cabling annulus, consisting of Seifert fibers of $Y$ ) and $A_{2}$ decompose $Y$ into two pieces $U_{1}$ and $U_{2}$, such that $U_{1}$ is a solid torus (which is $N_{*} \cap Y$ ) and $U_{2}$ is topologically $\partial M_{\mathrm{C}}$ times an interval. The above presentation for $\pi_{1}(Y)$ is obtained by applying the Van Kampen theorem associated to the splitting of $Y=U_{1} \cup_{A_{2}} U_{2}$ along $A_{2}$. We should note that as $|p|=1$, a longitude in $\partial N_{*}$ intersects $K$ geometrically exactly once. It is this curve pushed into $U_{1}$ which yields the element $\alpha$ and pushed into $U_{2}$ which yields $\beta$. Also because $|p|=1,\left\{\gamma_{\mathrm{C}}, \beta\right\}$ form a basis for $\pi_{1}\left(U_{2}\right)$. Therefore by Van Kampen, $\pi_{1}(Y)$ is generated by $\alpha, \beta, \gamma_{\mathrm{C}}$ with relations $\alpha^{q}=\gamma_{\mathrm{C}}$ and $\gamma_{\mathrm{C}} \beta=\beta \gamma_{\mathrm{C}}$, which yields presentation (5) after canceling the element $\gamma_{\mathrm{C}}$. With a suitable choice of orientation for $\beta$ i.e. replacing $\beta$ by its inverse if necessary, we also have $\mu_{\mathrm{K}}=\alpha \beta$. See Figure $\square$ for an illustration when $q=3$.

By [15, Lemma 7.2], $N_{\mathrm{K}}(p q)$, which denotes the manifold obtained by Dehn surgery on $K$ in the solid torus $N$ with the slope $p q$, is homeomorphic to $L(q, p) \#\left(D^{2} \times S^{1}\right)$, and $M_{\mathrm{K}}(p q)$, which denotes the manifold obtained by Dehn filling of $M_{\mathrm{K}}$ with the slope $p q$, is homeomorphic to $L(q, p) \# M_{\mathrm{C}}(p / q)$ (where the meaning of the notation $M_{\mathrm{C}}(p / q)$ should be obvious). As $|p|=1, M_{\mathrm{C}}(p / q)$ is a homology sphere. By [20], $R\left(M_{\mathrm{C}}(p / q)\right) \subset R\left(M_{\mathrm{C}}\right)$ contains at least one irreducible representation $\rho_{\mathrm{C}}$. Note that the restriction of $\rho_{\mathrm{C}}$ on $\pi_{1}\left(\partial M_{\mathrm{C}}\right)$ is not contained in $\{I,-I\}$. That is, we have: $\rho_{\mathrm{C}}\left(\gamma_{\mathrm{C}}\right)=I$ and $\rho_{\mathrm{C}}(\beta) \neq \pm I$ (we may consider $\beta$ as the longitude $\lambda_{\mathrm{C}}$ of $\left.\pi_{1}\left(\partial M_{\mathrm{C}}\right)\right)$. If $q>2$, we can extend $\rho_{\mathrm{C}}$ to a curve of representations of $\pi_{1}\left(M_{\mathrm{K}}\right)$ with one dimensional characters. In fact for every $A \in S L_{2}(\mathbb{C})$, we may define $\rho_{\mathrm{A}} \in R\left(M_{\mathrm{K}}\right)$ as follows. On $\pi_{1}\left(M_{\mathrm{C}}\right)$, let $\rho_{\mathrm{A}}=\rho_{\mathrm{C}}$, so in particular $\rho_{\mathrm{A}}(\beta)=\rho_{\mathrm{C}}(\beta)$, define $\rho_{\mathrm{A}}(\alpha)=A B A^{-1}$ where $B$ is a fixed order $q$ matrix in $S L_{2}(\mathbb{C})$. It's routine to check that $\rho_{\mathrm{A}}$ is well defined, the trace of $\rho_{\mathrm{A}}\left(\mu_{\mathrm{K}}\right)=A B A^{-1} \rho_{\mathrm{C}}(\beta)$ varies as $A$ runs over $S L_{2}(\mathbb{C})$, and $\rho_{\mathrm{A}}\left(\gamma_{\mathrm{K}}\right)=I$. Hence we get a curve in $X\left(M_{\mathrm{K}}\right)$ whose restriction on $\partial M_{\mathrm{K}}$ is one-dimensional, and moreover this curve generates $-1+y x^{q}$ as a factor of $A_{\mathrm{K}}(x, y)$ if $p=1$ or generates $-x^{q}+y$ as a factor of $A_{\mathrm{K}}(x, y)$ if $p=-1$. 


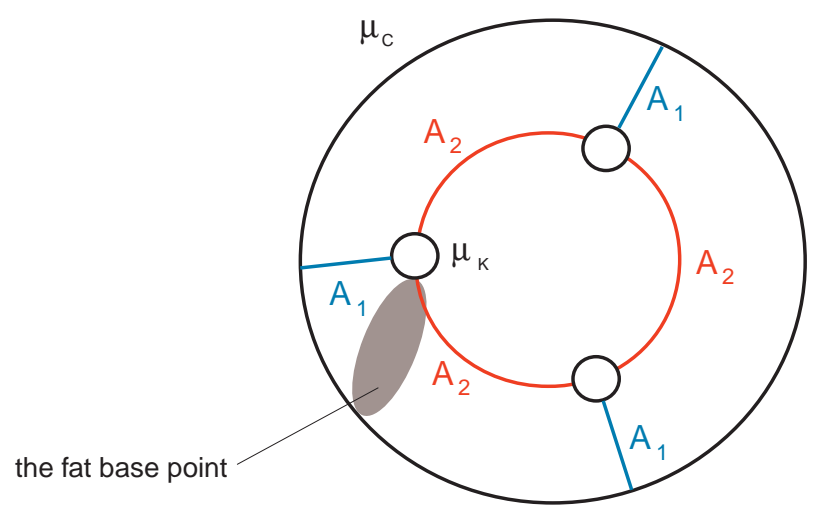

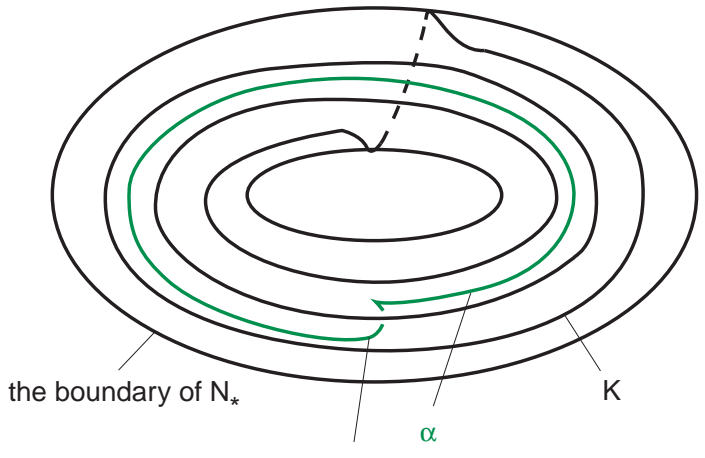

the base point

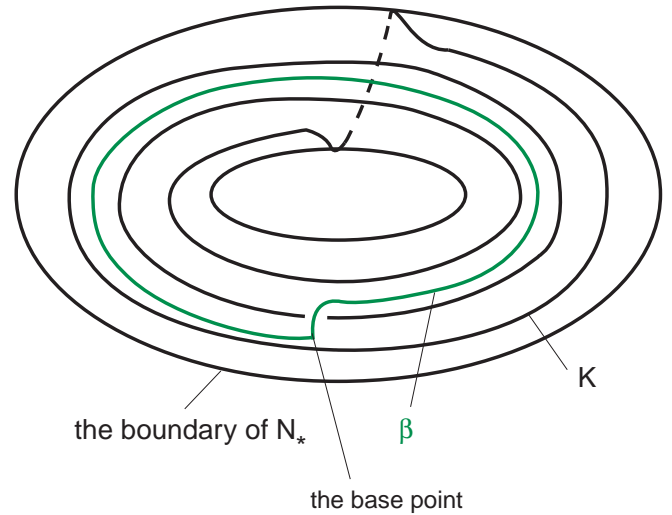

the base point

Figure 1: Illustration of the cross section $D \cap Y$ of $Y$ and the elements $\alpha$ and $\beta$ of $\pi_{1}(Y)$ when $q=3$.

We now show that $1+y x^{q}$ is a factor of $A_{\mathrm{K}}(x, y)$ if $p=1$ or $x^{q}+y$ is a factor of $A_{\mathrm{K}}(x, y)$ if $p=-1$, for all $q \geq 2$. For the representation $\rho_{\mathrm{C}}$ given in the last paragraph, defined $\rho_{\mathrm{C}}^{\epsilon} \in R\left(M_{\mathrm{C}}\right)$ by

$$
\rho_{\mathrm{C}}^{\epsilon}(\gamma)=\epsilon(\gamma) \rho_{\mathrm{C}}(\gamma) \text {, for } \gamma \in \pi_{1}\left(M_{\mathrm{C}}\right)
$$

where $\epsilon$ is the onto homomorphism $\epsilon: \pi_{1}\left(M_{\mathrm{C}}\right) \rightarrow\{I,-I\}$. As $\mu_{\mathrm{C}}$ is a generator of $H_{1}\left(M_{\mathrm{C}} ; \mathbb{Z}\right), \rho_{\mathrm{C}}^{\epsilon}\left(\mu_{\mathrm{C}}\right)=$ $-\rho_{\mathrm{C}}\left(\mu_{\mathrm{C}}\right)$. Similarly as $\lambda_{\mathrm{C}}$ is trivial in $H_{1}\left(M_{\mathrm{C}} ; \mathbb{Z}\right), \rho_{\mathrm{C}}^{\epsilon}\left(\lambda_{\mathrm{C}}\right)=\rho_{\mathrm{C}}\left(\lambda_{\mathrm{C}}\right)$. Hence $\rho_{\mathrm{C}}^{\epsilon}\left(\gamma_{\mathrm{C}}\right)=\rho_{\mathrm{C}}^{\epsilon}\left(\lambda_{\mathrm{C}}^{q} \mu_{\mathrm{C}}^{p}\right)=$ $-\rho_{\mathrm{C}}\left(\gamma_{\mathrm{C}}\right)=-I$. We can now extend $\rho_{\mathrm{C}}^{\epsilon}$ to $\rho_{\mathrm{A}}^{\epsilon}$ over $M_{\mathrm{K}}$ similarly as for $\rho_{\mathrm{C}}$ to $\rho_{\mathrm{A}}$, only this time we choose $B$ as a fixed order $2 q$ matrix in $S L_{2}(\mathbb{C})$, so that the trace of $\rho_{\mathrm{A}}^{\epsilon}\left(\mu_{\mathrm{K}}\right)=A B A^{-1} \rho_{\mathrm{C}}(\beta)$ varies as $A$ runs over $S L_{2}(\mathbb{C})$, and $\rho_{\mathrm{A}}^{\epsilon}\left(\gamma_{\mathrm{K}}\right)=-I$. The existence of the factor of $A_{\mathrm{K}}(x, y)$ that we set to prove now follows. This completes the proof of Claim 2.11.

We lastly prove Claim 2.12. Given a balanced-irreducible factor $f_{0}(x, y)$ of $A_{\mathrm{K}}(x, y)$, let $X_{0}$ be an irreducible component of $X^{*}\left(M_{\mathrm{K}}\right)$ over $\mathbb{C}$ which produces $f_{0}(x, y)$. Let $X_{0}^{\mathrm{Y}}$ and $X_{0}^{\mathrm{C}}$ be the Zariski closure of the restriction of $X_{0}$ on $Y$ and $M_{\mathrm{C}}$ respectively. Note that each of $X_{0}^{\mathrm{Y}}$ and $X_{0}^{\mathrm{C}}$ is irreducible. Also $X_{0}^{\mathrm{Y}}$ is at least one dimensional since its restriction on $\partial M_{\mathrm{K}}$ is one dimensional. If $X_{0}^{\mathrm{Y}}$ does not contain irreducible characters, then the restriction of $X_{0}^{\mathrm{Y}}$ on $\partial M_{\mathrm{C}}$ is also one dimensional. So the restriction of $X_{0}^{\mathrm{C}}$ on $\partial M_{\mathrm{C}}$ is one dimensional and $X_{0}$ is an abelian extension of $X_{0}^{\mathrm{C}}$ (since the character of a reducible representation is also the character of an abelian representation). It follows that $X_{0}^{\mathrm{C}}$ cannot be the trivial component in $X\left(M_{\mathrm{C}}\right)$ for otherwise $X_{0}$ woulde be the trivial component of $X\left(M_{\mathrm{K}}\right)$. Hence $X_{0}^{\mathrm{C}} \in X^{*}\left(M_{\mathrm{C}}\right)$ and $X_{0}$ is its abelian extension, which means that $f_{0}(x, y)$ is a factor of $\operatorname{Ext}^{q}\left[A_{\mathrm{C}}(\bar{x}, \bar{y})\right]$.

Hence we may assume that $X_{0}^{\mathrm{Y}}$ contains irreducible characters. Then, as noted before, we have $\rho\left(\gamma_{\mathrm{K}}\right)=\epsilon I$ for each $\rho$ with $\chi_{\rho} \in X_{0}^{\mathrm{Y}}$. If $\epsilon=-1$, then $1+x^{p q} y$ or $x^{p q}+y$ is the factor contributed by $X_{0}$ to $A_{\mathrm{K}}(x, y)$ 
corresponding to $p$ is positive or negative respectively. That is, $f_{0}(x, y)$ is a factor of $F_{(p, q)}(x, y)$. Hence we may assume that $\epsilon=1$. It follows that $X_{0}^{\mathrm{Y}}$ is a positive dimensional component of $N_{\mathrm{K}}(p q)$. Therefore $q>2$ (because $N_{\mathrm{K}}(p q)=L(q, p) \#\left(D^{2} \times S^{1}\right)$ ) and $X_{0}$ contributes the factor $-1+x^{p q} y$ or $-x^{p q}+y$ to $A_{\mathrm{K}}(x, y)$ corresponding to $p$ is positive or negative respectively. That is, $f_{0}$ is a factor of $F_{(p, q)}(x, y)$. This proves Claim 2.12 and also completes the proof of the theorem.

Perhaps we should note that in the above proof consistent choice of base points can be made for all the relevant manifolds such as $M_{\mathrm{K}}, M_{\mathrm{C}}, Y, A_{2}, U_{1}, U_{2}, \partial M_{\mathrm{C}}, \partial M_{\mathrm{K}}, N_{\mathrm{K}}(p q), M_{\mathrm{C}}(p / q), M_{\mathrm{K}}(p q)$ so that their fundamental groups are all well defined as relevant subgroups or quotient groups. In fact we can choose a simply connected region in $D \cap Y$ (as shown in Figure 1) so that its intersection with each relevant manifold listed above is a simply connected region which is served as the 'fat base point' of that manifold. $\diamond$

Example 2.13. Let $C$ be the figure 8 knot. Its $A$-polynomial is $A_{\mathrm{C}}(x, y)=x^{4}+\left(-1+x^{2}+2 x^{4}+x^{6}-\right.$ $\left.x^{8}\right) y+x^{4} y^{2}$ ([8, Appendix]). If $K$ is the $(p, 2)$-cable over $C, p>0$, then

$$
\begin{aligned}
A_{\mathrm{K}}(x, y) & =\operatorname{Red}\left[F_{(p, 2)}(x, y) \operatorname{Res} \bar{y}\left(A_{\mathrm{C}}\left(x^{2}, \bar{y}\right), \bar{y}^{2}-y\right)\right] \\
& =\left(1+x^{2 p} y\right)\left[x^{16}+\left(-1+2 x^{4}+3 x^{8}-2 x^{12}-6 x^{16}-2 x^{20}+3 x^{24}+2 x^{28}-x^{32}\right) y+x^{16} y^{2}\right] .
\end{aligned}
$$

If $K$ is the $(p, 3)$-cable over $C, p>0$, then

$$
\begin{aligned}
A_{\mathrm{K}}(x, y)= & \operatorname{Red}\left[F_{(p, 3)}(x, y) \operatorname{Res} \bar{y}\left(A_{\mathrm{C}}\left(x^{3}, \bar{y}\right), \bar{y}^{3}-y\right)\right] \\
= & \left(-1+x^{6 p} y^{2}\right)\left[x^{36}+\left(-1+3 x^{6}+3 x^{12}-8 x^{18}-12 x^{24}+6 x^{30}+20 x^{36}+6 x^{42}-12 x^{48}\right.\right. \\
& \left.\left.-8 x^{54}+3 x^{60}+3 x^{66}-x^{72}\right) y+x^{36} y^{2}\right]
\end{aligned}
$$

Finally we would like to give an explicit formula for the $A$-polynomials of iterated torus knots. Let

$$
K=\left[\left(p_{1}, q_{2}\right),\left(p_{2}, q_{2}\right), \ldots,\left(p_{n}, q_{n}\right)\right]
$$

be an $n$-th iterated torus knot, i.e. $T\left(p_{n}, q_{n}\right)$ is a nontrivial torus knot and when $n>1$, for each $i, n>i \geq 1,\left[\left(p_{i}, q_{i}\right),\left(p_{i+1}, q_{i+1}\right), \ldots,\left(p_{n}, q_{n}\right)\right]$ is a satellite knot with $\left[\left(p_{i+1}, q_{i+1}\right), \ldots,\left(p_{n}, q_{n}\right)\right]$ as a companion knot and with $T\left(p_{i}, q_{i}\right), q_{i}>1$, as a pattern knot lying in the trivial solid torus $V$ as a $\left(p_{i}, q_{i}\right)$-cable with winding number $q_{i}$, where $\left|p_{i}\right|$ may be less than $q_{i}$ and $\left|p_{i}\right|=1$ is also allowed.

Corollary 2.14. Let $K=\left[\left(p_{1}, q_{1}\right),\left(p_{2}, q_{2}\right), \ldots,\left(p_{n}, q_{n}\right)\right]$ be an iterated torus knot. If for each $1 \leq i<n$, $q_{i}$ is odd, then

$$
A_{K}(x, y)=F_{\left(p_{1}, q_{1}\right)}(x, y) F_{\left(p_{2}, q_{2}\right)}\left(x^{q_{1}^{2}}, y\right) F_{\left(p_{3}, q_{3}\right)}\left(x^{q_{1}^{2} q_{2}^{2}}, y\right) \cdots F_{\left(p_{n}, q_{n}\right)}\left(x^{q_{1}^{2} q_{2}^{2} \cdots q_{n-1}^{2}}, y\right)
$$

and if for some $1 \leq i<n, q_{i}$ is even, we let $m$ be the smallest such integer, then

$$
\begin{aligned}
A_{K}(x, y)= & F_{\left(p_{1}, q_{1}\right)}(x, y) F_{\left(p_{2}, q_{2}\right)}\left(x^{q_{1}^{2}}, y\right) F_{\left(p_{3}, q_{3}\right)}\left(x^{q_{1}^{2} q_{2}^{2}}, y\right) \cdots F_{\left(p_{m}, q_{m}\right)}\left(x^{q_{1}^{2} q_{2}^{2} \cdots q_{m-1}^{2}}, y\right) \\
& G_{\left(p_{m+1}, q_{m+1}\right)}\left(x^{q_{1}^{2} q_{2}^{2} \cdots q_{m}^{2}}, y\right) G_{\left(p_{m+2}, q_{m+2}\right)}\left(x^{q_{1}^{2} q_{2}^{2} \cdots q_{m+1}^{2}}, y\right) \cdots G_{\left(p_{n}, q_{n}\right)}\left(x^{q_{1}^{2} q_{2}^{2} \cdots q_{n-1}^{2}}, y\right)
\end{aligned}
$$

Remark 2.15. (1) In the $A$-polynomial given in the corollary, each

$$
F_{\left(p_{i}, q_{i}\right)}\left(x^{q_{1}^{2} q_{2}^{2} \cdots q_{i-1}^{2}}, y\right) \text { or } G_{\left(p_{i}, q_{i}\right)}\left(x^{q_{1}^{2} q_{2}^{2} \cdots q_{i-1}^{2}}, y\right)
$$

is a nontrivial polynomial even when $\left|p_{i}\right|=1$.

(2) The polynomial expression for $A_{K}(x, y)$ given in the corollary has no repeated factors.

(3) The boundary slopes detected by $A_{K}(x, y)$ are precisely the following $n$ integer slopes:

$$
p_{1} q_{1}, p_{2} q_{2} q_{1}^{2}, p_{3} q_{3} q_{1}^{2} q_{2}^{2}, \ldots, p_{n} q_{n} q_{1}^{2} q_{2}^{2} \cdots q_{n-1}^{2} \text {. }
$$

Proof. The proof goes by induction on $n$ applying Theorem 2.10. When $n=1$, the proposition holds obviously. Suppose for $n-1 \geq 1$ the proposition holds. Note that $K$ has $C=\left[\left(p_{2}, q_{2}\right), \ldots,\left(p_{n}, q_{n}\right)\right]$ as a companion knot and $P=T\left(p_{1}, q_{1}\right)$ as the corresponding pattern knot (which maybe a trivial knot in $S^{3}$, which occurs exactly when $\left.\left|p_{1}\right|=1\right)$. By induction, the $A$-polynomial $A_{\mathrm{C}}(x, y)$ of $C$ is of the corresponding form as described by the corollary. Now applying Theorem 2.10 one more time to the pair $(C, P)$ we see that the corollary holds. We omit the routine details. 
For instance the A-polynomial of the $(r, s)$-cable over the $(p, q)$-torus knot is

$$
A(x, y)= \begin{cases}F_{(r, s)}(x, y) F_{(p, q)}\left(x^{s^{2}}, y\right), & \text { if } s \text { is odd } \\ F_{(r, s)}(x, y) G_{(p, q)}\left(x^{s^{2}}, y\right), & \text { if } s \text { is even. }\end{cases}
$$

\section{Proof of Theorem 1.1}

Suppose that $K$ is a knot in $S^{3}$ with the same knot Floer homology and the same A-polynomial as a given torus knot $T(p, q)$. Our goal is to show that $K=T(p, q)$.

By (3) and Lemma 2.4, $K$ is not a hyperbolic knot. So $K$ is either a torus knot or a satellite knot.

Lemma 3.1. Suppose that $T(r, s)$ is a torus knot whose A-polynomial divides that of $T(p, q)$ and whose Alexander polynomial divides that of $T(p, q)$, then $T(r, s)=T(p, q)$.

Proof. Since $A_{T(r, s)}(x, y) \mid A_{T(p, q)}(x, y)$, from the formula (3), we have $r s=p q$. From the condition

$$
\Delta_{T(r, s)}(t)=\frac{\left(t^{r s}-1\right)(t-1)}{\left(t^{r}-1\right)\left(t^{s}-1\right)} \mid \Delta_{T(p, q)}(t)=\frac{\left(t^{p q}-1\right)(t-1)}{\left(t^{p}-1\right)\left(t^{q}-1\right)}
$$

we have

$$
\left(t^{p}-1\right)\left(t^{q}-1\right) \mid\left(t^{r}-1\right)\left(t^{s}-1\right) .
$$

Now if $T(r, s) \neq T(p, q)$, then either $q>s$ or $|p|>|r|$. If $q>s$, then from (6) we must have $q \mid r$. By our convention for parameterizing torus knots, $|p|>q \geq 2$. Thus we must also have $p \mid r$. But $p$ and $q$ are relatively prime, we have $p q \mid r$, which contradicts the early conclusion $r s=p q$. If $|p|>|r|$, again by our convention $|r|>s \geq 2$, we see that $\left(t^{p}-1\right)$ does not divide $\left(t^{r}-1\right)\left(t^{s}-1\right)$, and so (6) cannot hold. This contradiction completes the proof of the lemma. $\diamond$

By Lemma 3.1 we see that if $K$ is a torus knot, then $K=T(p, q)$.

We are going to show that it is impossible for $K$ to be a satellite knot, which will consist of the rest of the proof of Theorem 1.1. Suppose that $K$ is a satellite knot. We need to derive a contradiction from this assumption. Let $C$ and $P$ be a pair of associated companion knot and pattern knot to $K$, and let $w$ be the winding number of $P$ in its defining solid torus $V$ (recall the definition of a satellite knot given in Section 2). As $T(p, q)$ is a fibred knot, $K$ is also fibred. According to [7, Corollary 4.15 and Proposition 8.23], each of $C$ and $P$ is a fibred knot in $S^{3}, w \geq 1$, and the Alexander polynomials of these knots satisfy the equality

$$
\Delta_{\mathrm{K}}(t)=\Delta_{\mathrm{C}}\left(t^{w}\right) \Delta_{\mathrm{P}}(t)
$$

We may choose $C$ such that $C$ is itself not a satellite knot, and thus is either a hyperbolic knot or a torus knot.

Lemma 3.2. The companion knot $C$ cannot be a hyperbolic knot.

Proof. Suppose that $C$ is hyperbolic. Then $A_{\mathrm{C}}(\bar{x}, \bar{y})$ contains a balanced-irreducible factor $f_{\mathrm{C}}(\bar{x}, \bar{y})$ whose Newton polygon detects at least two distinct boundary slopes of $C$. As $w \geq 1$, by Proposition 2.8, $f_{\mathrm{C}}(\bar{x}, \bar{y})$ extends to a balanced factor $f_{\mathrm{K}}(x, y)$ of $A_{\mathrm{K}}(x, y)=A_{T(p, q)}(x, y)$. Moreover from the relation (44) we see that the Newton polygon of $f_{\mathrm{K}}(x, y)$ detects at least two distinct boundary slopes of $K$. But clearly the Newton polygon of $A_{\mathrm{K}}(x, y)=A_{T(p, q)}(x, y)$ only detects one boundary slope. We arrive at a contradiction. $\diamond$

So $C=T(r, s)$ is a torus knot.

Lemma 3.3. The pattern knot $P$ of $K$ cannot be the unknot.

Proof. Suppose otherwise that $P$ is the unknot. Then as noted in [19] the winding number $w$ of $P$ in its defining solid torus $V$ is larger than 1. Equality (77) becomes $\Delta_{K}(t)=\Delta_{C}\left(t^{w}\right)$ for some integer $w>1$. On the other hand it is easy to check that the degrees of the leading term and the second term of $\Delta_{T(p, q)}(t)$ differ by 1 and thus $\Delta_{\mathrm{K}}(t)=\Delta_{T(p, q)}(t)$ cannot be of the form $\Delta_{\mathrm{C}}\left(t^{w}\right), w>1$. This contradiction completes the proof. 
If $w=1$, then by Proposition 2.8, $A_{\mathrm{C}}(x, y)=A_{T(r, s)}(x, y)$ divides $A_{\mathrm{K}}(x, y)=A_{T(p, q)}(x, y)$, and by (7) $\Delta_{\mathrm{C}}(t)=\Delta_{T(r, s)}(t)$ divides $\Delta_{\mathrm{K}}(t)=\Delta_{T(p, q)}(t)$. Hence by Lemma 3.1 we have $C=T(r, s)=T(p, q)$. By Lemma 3.3 $P$ is a non-trivial knot. Hence from formula (77), we see that the genus of $C=T(p, q)$ is less than that of $K$. But the genus of $K$ is equal to that of $T(p, q)$. We derive a contradiction.

Hence $w>1$. By Lemma 2.7. $A_{\mathrm{P}}(x, y)$ divides $A_{\mathrm{K}}(x, y)$. Now if $P$ is itself a satellite knot with its own companion knot $C_{1}$ and pattern knot $P_{1}$. Then again each of $C_{1}$ and $P_{1}$ is a fibred knot and the winding number $w_{1}$ of $P_{1}$ with respect to $C_{1}$ is larger than zero. Arguing as above, we see that $C_{1}$ may be assumed to be a torus knot and that $w_{1}>1$. Also we have

$$
\Delta_{\mathrm{K}}(t)=\Delta_{\mathrm{C}}\left(t^{w}\right) \Delta_{\mathrm{C}_{1}}\left(t^{w_{1}}\right) \Delta_{\mathrm{P}_{1}}(t)
$$

from which we see that $P_{1}$ cannot be the trivial knot just as in the proof of Lemma 3.3 .

So after a finitely many such steps (the process must terminate by [27]), we end up with a pattern knot $P_{m}$ for $P_{m-1}$ such that $P_{m}$ is nontrivial but is no longer a satellite knot. Thus $P_{m}$ is either a hyperbolic knot or a torus knot. By Lemma 2.4 and Lemma 2.7 $P_{m}$ cannot be hyperbolic. Hence $P_{m}$ is a torus knot. Again because $A_{\mathrm{P}_{m}}(x, y)$ divides $A_{\mathrm{K}}(x, y)=A_{T(p, q)}(x, y)$ and $\Delta_{\mathrm{P}_{m}}(t)$ divides $\Delta_{\mathrm{K}}(t)=\Delta_{T(p, q)}(t)$, we have $P_{m}=T(p, q)$ by Lemma 3.1. But once again we would have $g(T(p, q))<g(T(p, q))$. This gives a final contradiction.

\section{The knots $k(l, m, n, p)$}

In [12] a family of hyperbolic knots $k(l, m, n, p)$ in $S^{3}$ (where at least one of $p$ and $n$ has to be zero) was constructed such that each knot in the family admits one (and only one) half-integral toroidal surgery. To be hyperbolic, the following restrictions on the values for $l, m, n, p$ are imposed:

$$
\begin{aligned}
& \text { If } p=0 \text {, then } l \neq 0, \pm 1, m \neq 0,(l, m) \neq(2,1),(-2,-1),(m, n) \neq(1,0),(-1,1) \text {; } \\
& \text { If } n=0 \text {, then } l \neq 0, \pm 1, m \neq 0,1,(l, m, p) \neq(-2,-1,0),(2,2,1) .
\end{aligned}
$$

From now on we assume that any given $k(l, m, n, p)$ is hyperbolic, i.e. $l, m, n, p$ satisfy the above restrictions.

The half-integral toroidal slope $r=r(l, m, n, p)$ of $k(l, m, n, p)$ was explicitly computed in 13, Proposition 5.3] as given below:

$$
r= \begin{cases}l(2 m-1)(1-l m)+n(2 l m-1)^{2}-\frac{1}{2}, & \text { when } p=0 \\ l(2 m-1)(1-l m)+p(2 l m-l-1)^{2}-\frac{1}{2}, & \text { when } n=0\end{cases}
$$

It turns out that $k(l, m, n, p)$ are the only hyperbolic knots in $S^{3}$ which admit non-integral toroidal surgeries.

Theorem 4.1. ([17]) If a hyperbolic knot $K$ in $S^{3}$ admits a non-integral toroidal surgery, then $K$ is one of the knots $k(l, m, n, p)$.

Proposition 4.2. The knots $k(l, m, n, p)$ have the following properties:

(a) $k(l, m, n, 0)$ is the mirror image of $k(-l,-m, 1-n, 0)$.

(b) $k(l, m, 0, p)$ is the mirror image of $k(-l, 1-m, 0,1-p)$.

(c) $k(l, \pm 1, n, 0)=k(-l \pm 1, \pm 1, n, 0)$.

(d) $k(2,-1, n, 0)=k(-3,-1, n, 0)=k(2,2,0, n)$.

Proof. This follows from [12, Proposition 1.4]. 

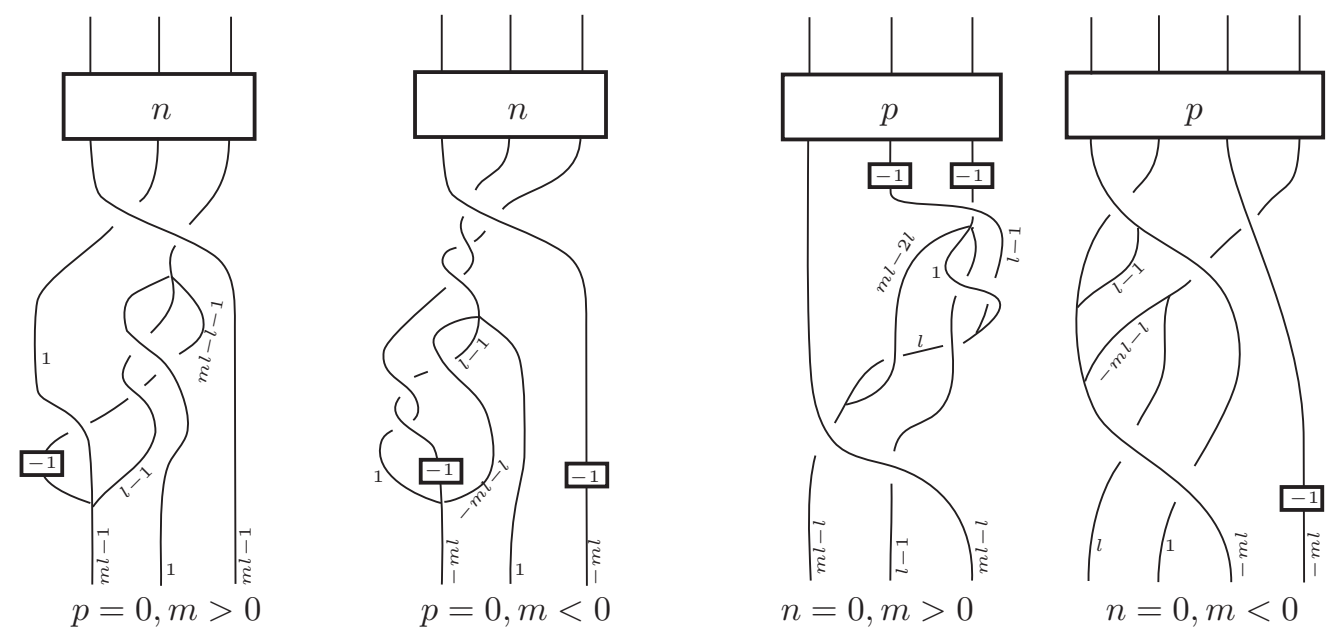

Figure 2: The braid whose closure is $k(l, m, n, p)$, where $l>0$.

Explicit closed braid presentations for the knots $k(l, m, n, p)$ are given in 13 . Figure 2 shows the braids whose closure are $k(l, m, n, p)$. The left two pictures are a reproduction of [13, Fig. 12], but the right two pictures are different from [13, Fig. 13]. Here an arc with label $s$ means $s$ parallel strands, and a box with label $t$ means $t$ positive full-twists when $t>0$ and $|t|$ negative full-twists when $t<0$. We only give the picture for the case $l>0$, since the case $l<0$ can be treated by applying Proposition 4.2.

Proposition 4.3. Suppose that $l>0$, let

$$
N= \begin{cases}2 m l-1, & \text { if } p=0, n \neq 0, m>0, \\ -2 m l+1, & \text { if } p=0, n \neq 0, m<0, \\ 2 m l-l-1, & \text { if } n=0, m>0, \\ -2 m l+l+1, & \text { if } n=0, m<0 .\end{cases}
$$

Then the genus of $k(l, m, n, 0)$ is

$$
g=|n| \frac{N(N-1)}{2}+ \begin{cases}m^{2} l^{2}-m \frac{l(l+5)}{2}+l+1, & \text { if } m>0, n \leq 0, \\ -m^{2} l^{2}+m \frac{l(l+1)}{2}-l+1, & \text { if } m>0, n>0, \\ m^{2} l^{2}-m \frac{l(l-1)}{2}, & \text { if } m<0, n \leq 0, \\ -m^{2} l^{2}+m \frac{l(l+3)}{2}, & \text { if } m<0, n>0,\end{cases}
$$

and the genus of $k(l, m, 0, p)$ is

$$
g=|p| \frac{N(N-1)}{2}+ \begin{cases}m^{2} l^{2}-m \frac{l(l+5)}{2}+l+1, & \text { if } m>0, p \leq 0, \\ -m^{2} l^{2}+m \frac{l(l+1)}{2}+1, & \text { if } m>0, p>0, \\ m^{2} l^{2}-m \frac{l(l-1)}{2}, & \text { if } m<0, p \leq 0, \\ -m^{2} l^{2}+m \frac{l(l+3)}{2}-l, & \text { if } m<0, p>0 .\end{cases}
$$

Proof. In [13, it is noted that $k(l, m, n, p)$ is the closure of a positive or negative braid. Hence it is fibered, and the genus can be computed by the formula

$$
g(k)=\frac{C-N+1}{2}
$$

where $C$ is the crossing number in the positive or negative braid, and $N$ is the braid index.

When $p=0, m>0, n \leq 0$, the braid is a negative braid, $N=2 m l-1$,

$$
C=-n(2 m l-1)(2 m l-2)+m l(m l-1)+m l-2+l(m l-l-1)+(m l-l-1)(m l-l-2),
$$




$$
g=-n(2 m l-1)(m l-1)+m^{2} l^{2}-m \frac{l(l+5)}{2}+l+1
$$

When $p=0, m>0, n>0$, we can cancel all the negative crossings in the braid to get a positive braid of index $N=2 m l-1$. We have

$$
C=n(2 m l-1)(2 m l-2)-(m l(m l-1)+m l-2+l(m l-l-1)+(m l-l-1)(m l-l-2)),
$$

SO

$$
g=n(2 m l-1)(m l-1)-m^{2} l^{2}+m \frac{l(l+1)}{2}-l+1
$$

The computation for other cases are similar. $\diamond$

Using Proposition 4.3 and Proposition 4.2 (a)(b), we can compute the genus of $k(l, m, n, p)$ when $l<0$. For example, when $p \leq 0$, the genus of $k(l, m, 0, p)$ is

$$
g= \begin{cases}-p \frac{(2 m l-l-1)(2 m l-l-2)}{2}+m^{2} l^{2}-m \frac{l(l+5)}{2}+l+1, & \text { if } l>0, m>0, \\ -p \frac{(-2 m l+l+1)(-2 m l+l)}{2}+m^{2} l^{2}-m \frac{l(l-1)}{2}, & \text { if } l>0, m<0, \\ -p \frac{(-2 m l+l+1)(-2 m l+l)}{2}+m^{2} l^{2}-m \frac{l(l-1)}{2}, & \text { if } l<0, m>0, \\ -p \frac{(2 m l-l-1)(2 m l-l-2)}{2}+m^{2} l^{2}-m \frac{l(l+5)}{2}+l+2, & \text { if } l<0, m<0 .\end{cases}
$$

The $r$-surgery on the knot $J=k(l, m, n, p)$ was explicitly given in [12] which is the double branched cover of $S^{3}$ with the branched set in $S^{3}$ being a link shown in Figure 3 . From the tangle decomposition of the branched link one can see that $M_{\mathrm{J}}(r)$ is a graph manifold obtained by gluing two Seifert fibred spaces, each over a disk with two cone points, together along their torus boundaries. For our purpose, we need to give a more detailed description of the graph manifold $M_{\mathrm{J}}(r)$ as follows.

Let $(B, t)$ denote a two string tangle, i.e. $B$ is a 3 -ball and $t$ is a pair of disjoint properly embedded arcs in $B$. Here we may assume that $B$ is the unit 3 -ball in the $x y z$-space $\mathbb{R}^{3}$ (with the $x y$-plane horizontal) and that the four endpoints of $t$ lies in the lines $z=y, x=0$ and $z=-y, x=0$. Let $D$ be the unit disk in $B$ which is the intersection of $B$ with the $y z$-plane. Then the four endpoints of $t$ divides $\partial D$ into four arcs, naturally named the east, west, north, south arcs. The denominator closure of $(B, t)$ is the link in $S^{3}$ obtained by capping off $t$ with the east and west arcs, and the numerator closure of $(B, t)$ is the link in $S^{3}$ obtained by capping off $t$ with the north and south arcs.

Let $\left(B_{i}, t_{i}\right), i=1,2$, be the two tangles shown in Figure 3 and let $X_{i}$ be the double branched cover of $\left(B_{i}, t_{i}\right)$. The denominator closure of $\left(B_{1}, t_{1}\right)$ is the twisted knot of type $(2, p)$ (which is the trivial knot when $p=0,1$, the trefoil knot when $p=-1$ ) and therefore the double branched cover of $S^{3}$ over the link is the lens space of order $|2 p-1|$. The numerator closure of $\left(B_{1}, t_{1}\right)$ gives a composite link in $S^{3}$ and in fact the composition of two nontrivial rational links corresponding to the rational numbers $-l$ and $(-\operatorname{lm}(2 p-1)+p l+2 p-1) /(-m(2 p-1)+p)$.

The double branched cover of the east arc (as well as the west arc) is a simple closed essential curve in $\partial X_{1}$, which we denote by $\mu_{1}$, with which Dehn filling of $X_{1}$ is a lens space of order $|2 p-1|$. The double branched cover of the north arc (as well as the south arc) is a Seifert fiber of $X_{1}$, which we denote by $\sigma_{1}$, with which Dehn filling of $X_{1}$ is a connected sum of two nontrivial lens spaces of orders $|-l|$ and $|-\operatorname{lm}(2 p-1)+p l+2 p-1|$.

Similarly the numerator closure of $\left(B_{2}, t_{2}\right)$ is the twisted knot of type $(2, n)$ and the denominator closure of $\left(B_{2}, t_{2}\right)$ is a composite link of two nontrivial rational links corresponding to the rational numbers -2 and $(2(2 n-1)(m-1)+4 n-1) /(2 n-1)(m-1)+n)$. So the double branched cover of the north arc of $\left(B_{2}, t_{2}\right)$ is a simple closed essential curve in $\partial X_{2}$, denoted $\mu_{2}$, with which Dehn filling of $X_{2}$ is a lens space of order $|2 n-1|$, and the double branched cover of the west arc is a Seifert fiber of $X_{2}$, denoted by $\sigma_{2}$, with which Dehn filling is a connected sum of two nontrivial lens spaces of orders 2 and $|2(2 n-1)(m-1)+4 n-1|$. 


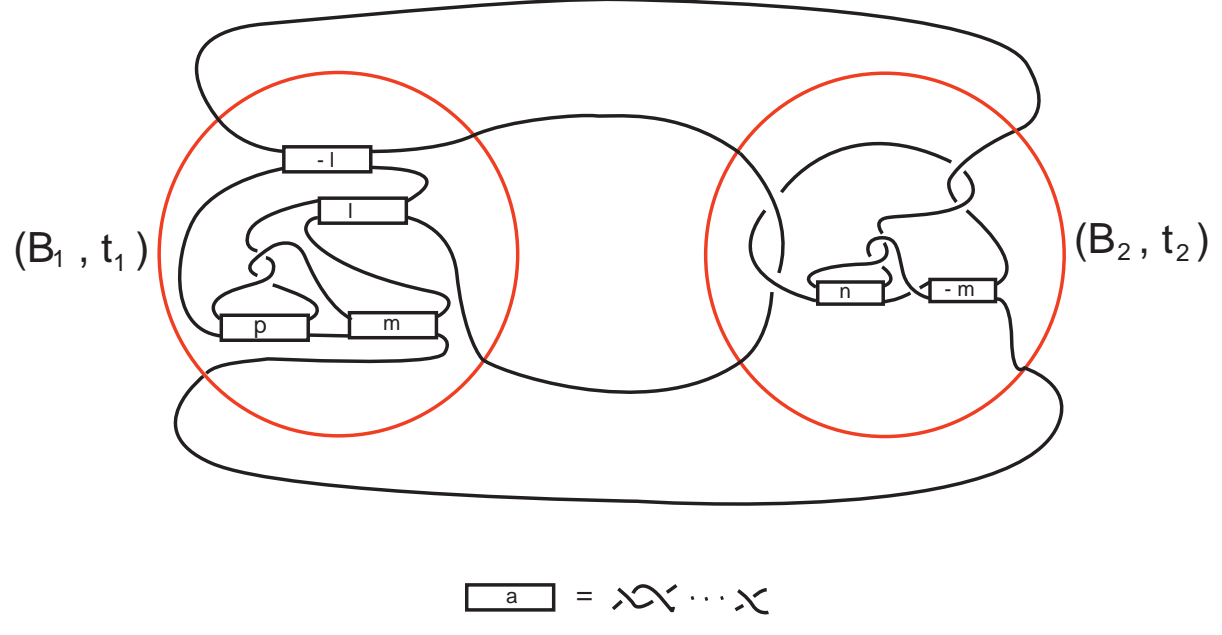

Figure 3: The tangle decomposition of the branched link in $S^{3}$ for the half-integral toroidal surgery

Finally $M_{\mathrm{J}}(r)$ is obtained by gluing $X_{1}$ and $X_{2}$ along their boundary tori such that $\mu_{1}$ is identified with $\sigma_{2}$ and $\sigma_{1}$ with $\mu_{2}$.

Note that when $p=0$ or $1, X_{1}$ is the exterior of a torus knot in $S^{3}$, and when $n=0$ or $1, X_{2}$ is the exterior of a $(2, a)$ torus knot in $S^{3}$.

Lemma 4.4. Let $J=k(l, m, n, p), r$ the unique half-integral toroidal slope of $J$ and $M_{J}$ the exterior of $J$. Up to isotopy, there is a unique closed orientable incompressible surface in $M_{J}(r)$, which is a torus.

Proof. As discussed above, $M_{\mathrm{J}}(r)$ is a graph manifold with the torus decomposition $M_{\mathrm{J}}(r)=X_{1} \cup X_{2}$. We just need to show that any connected closed orientable incompressible surface $S$ in $M_{\mathrm{J}}(r)$ is isotopic to $\partial X_{1}$. Suppose otherwise that $S$ is not isotopic to $\partial X_{1}$. As each $X_{i}$ does not contain closed essential surfaces, $S$ must intersect $\partial X_{1}$. We may assume that $F_{i}=S \cap X_{i}$ is incompressible and boundary incompressible, for each $i=1,2$. As $X_{i}$ is Seifert fibred, $F_{i}$ is either horizontal (i.e. consisting of Seifert fibres or vertical (transverse to Seifert fibres) in $X_{i}$, up to isotopy. So the boundary slope of $F_{i}$ is either $\sigma_{i}$ (when $F_{i}$ is horizontal) or is the rational longitude of $X_{i}$ (when $F_{i}$ is vertical). But $\sigma_{i}$ is identified with $\mu_{j}$, for $i=1,2,\{i, j\}=\{1,2\}$. So the boundary slope of $F_{i}$ must be the rational longitude of $X_{i}$ for each $i$. It follows that $H_{1}\left(M_{\mathrm{J}}(r) ; \mathbb{Z}\right)$ is infinite, yielding a contradiction. $\diamond$

Lemma 4.5. For each $J=k(l, m, n, p)$, its meridian slope is not a boundary slope.

Proof. If the meridian slope of $J$ is a boundary slope, then the knot exterior $M_{\mathrm{J}}$ contains a connected closed orientable incompressible surface $S$ of genus larger than one such that $S$ remains incompressible in any non-integral surgery, by [9, Theorem 2.0.3]. But by Lemma 4.4, $M_{\mathrm{J}}(r)$ does not contain any closed orientable incompressible surface of genus larger than one. This contradiction completes the proof. $\diamond$

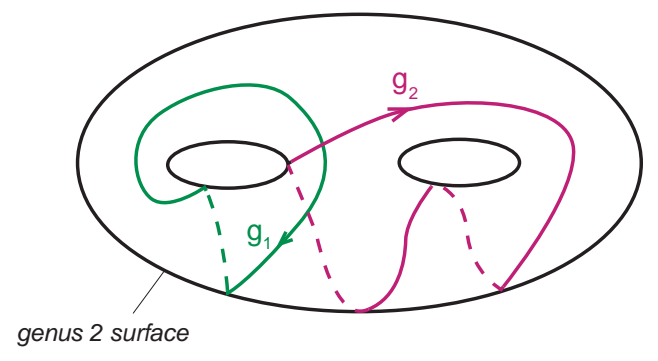

Figure 4: The elements $g_{1}$ and $g_{2}$ in $\partial H$

Lastly in this section we are going to show that $k\left(l_{*},-1,0,0\right)$ is a class of small knots. 
Let $H$ be a standard genus two handlebody in $S^{3}$ and let $g_{1}$ and $g_{2}$ be oriented loops in the surface $\partial H$ as shown in Figure 4. As observed in [2, Figure 8], a regular neighborhood of $g_{1} \cup g_{2}$ in $\partial H$ is a genus one Seifert surface of a trefoil knot. Under the basis $\left[g_{1}\right],\left[g_{2}\right]$, the monodromy of the trefoil is represented by the matrix

$$
\left(\begin{array}{cc}
0 & 1 \\
-1 & 1
\end{array}\right)
$$

From [13. Figure 11], the knot $k\left(l_{*},-1,0,0\right)$ has a knot diagram as shown in Figure 5 (a), which in turn can be embedded in the Seifert surface with the homology class $l_{*}\left[g_{1}\right]+\left(l_{*}+1\right)\left[g_{2}\right]$ as shown in Figure 5 (b). For any knot $J$ lying in the Seifert surface, an algorithm to classify all closed essential surfaces in the knot exterior of $J$ is given in [1, Theorem 10.1 (3)]. For simplicity, we only state the part that we need:

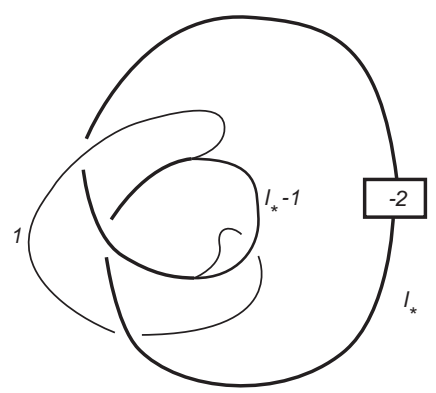

(a)

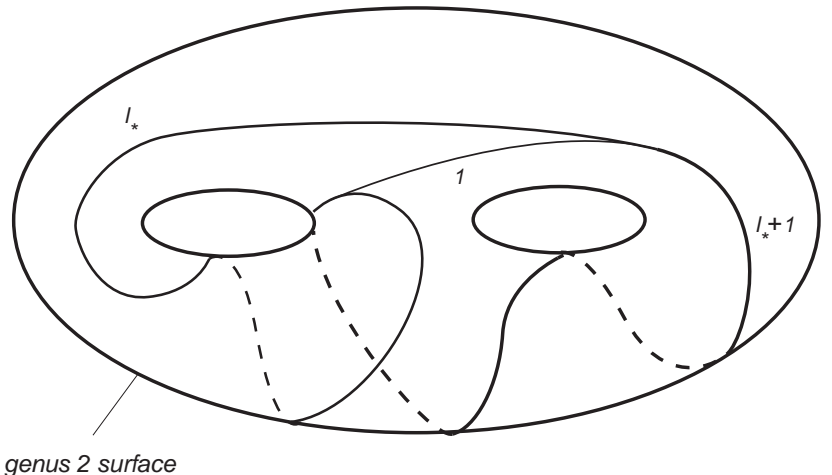

(b)

Figure 5: The knot $k\left(l_{*},-1,0,0\right)$

Theorem 4.6. Suppose that $J$ is a simple closed curve on the genus one Seifert surface of a trefoil knot in the homology class $a_{1}\left[g_{1}\right]+a_{2}\left[g_{2}\right]$. The slope $\frac{a_{1}}{a_{2}}$ has continued fraction expansion

$$
\frac{a_{1}}{a_{2}}=\left[b_{1}, \ldots, b_{k}\right]=b_{1}-\frac{1}{b_{2}-\frac{1}{\ddots-\frac{1}{b_{k}}}},
$$

where the coefficients alternate signs, $b_{i} \neq 0$ when $i \geq 2$, and $\left|b_{k}\right| \geq 2$. If $b_{1}=0$ and $b_{2}=-1$, then every closed essential surface in the complement of $L$ corresponds to a solution of the following equation:

$$
0=\sum_{i \in I}-b_{i}+\sum_{j \in J} b_{j}+ \begin{cases}0, & \text { if } 3 \in J, \\ -1, & \text { otherwise },\end{cases}
$$

where $I$ and $J$ are subsets of $\{3, \ldots, k\}$ each not containing consecutive integers and $3 \notin I \cap J$.

Lemma 4.7. Each $k\left(l_{*},-1,0,0\right)$ is a small knot. Namely, the exterior of $k\left(l_{*},-1,0,0\right)$ contains no closed essential surfaces.

Proof. In this case $a_{1}=l_{*}, a_{2}=l_{*}+1, \frac{a_{1}}{a_{2}}=\left[0,-1, l_{*}\right]$. Clearly, Equation (11) has no solution. So $k\left(l_{*},-1,0,0\right)$ is small. $\diamond$

\section{Information on A-polynomials of $k(l, m, n, p)$}

To obtain explicit expressions for the $A$-polynomials of the knots $k(l, m, n, p)$ could be a very tough task. But we can obtain some useful info about the $A$-polynomials of these knots without computing them explicitly. 
Lemma 5.1. Suppose a graph manifold $W$ is obtained by gluing two torus knot exteriors $X_{1}, X_{2}$ together, such that the meridian of $X_{i}$ is glued to the Seifert fiber of $X_{i+1}, i=1,2$, where $X_{3}=X_{1}$. Then $\pi_{1}(W)$ has no non-cyclic $S L_{2}(\mathbb{C})$ representations.

To prove this lemma, we will use the following well-known fact whose proof is elementary.

Lemma 5.2. Suppose that $A, B \in S L_{2}(\mathbb{C})$ are two commuting matrices, $A \neq \pm I$.

(i) If there exits $P \in S L_{2}(\mathbb{C})$ such that

$$
P A P^{-1}=\left(\begin{array}{cc}
\lambda & 0 \\
0 & \lambda^{-1}
\end{array}\right), \quad \text { for some } \lambda \in \mathbb{C} \backslash\{0, \pm 1\},
$$

then

$$
P B P^{-1}=\left(\begin{array}{cc}
\mu & 0 \\
0 & \mu^{-1}
\end{array}\right), \quad \text { for some } \mu \in \mathbb{C} \backslash\{0\} ;
$$

(ii) If there exits $P \in S L_{2}(\mathbb{C})$ such that

$$
P A P^{-1}= \pm\left(\begin{array}{ll}
1 & a \\
0 & 1
\end{array}\right), \quad \text { for some } a \in \mathbb{C} \backslash\{0\},
$$

then

$$
P B P^{-1}= \pm\left(\begin{array}{ll}
1 & b \\
0 & 1
\end{array}\right), \quad \text { for some } b \in \mathbb{C} .
$$

Proof of Lemma 5.1. As $W$ has cyclic homology group, it is equivalent to show that every $S L_{2}(\mathbb{C})$ representation of $\pi_{1}(W)$ is abelian.

We choose a base point of $W$ on the common boundary torus $T$ of $X_{1}, X_{2}$, then $\pi_{1}(T), \pi_{1}\left(X_{1}\right), \pi_{1}\left(X_{2}\right)$ are naturally the subgroups of $\pi_{1}(W)$. Suppose that $\rho: \pi_{1}(W) \rightarrow S L_{2}(\mathbb{C})$ is a representation.

Let $f_{i} \in \pi_{1}(T)$ represent the Seifert fiber of $X_{i}, i=1,2$. We first consider the case that one of $\rho\left(f_{1}\right), \rho\left(f_{2}\right)$, say $\rho\left(f_{1}\right)$, is in $\{ \pm I\}$ which is the center of $S L_{2}(\mathbb{C})$. Since $f_{1}$ represents the meridian of $X_{2}, f_{1}$ normally generates $\pi_{1}\left(X_{2}\right)$, hence $\rho\left(\pi_{1}\left(X_{2}\right)\right)$ is contained in $\{ \pm I\}$. Since $f_{2} \in \pi_{1}\left(X_{2}\right)$, we see that $\rho\left(f_{2}\right)$ is also in $\{ \pm I\}$, and thus for the same reason as just given $\rho\left(\pi_{1}\left(X_{1}\right)\right)$ is in $\{ \pm I\}$. Hence $\rho(\pi(W))$ is contained in $\{ \pm I\}$.

Now suppose that neither of $\rho\left(f_{1}\right), \rho\left(f_{2}\right)$ is in $\{ \pm I\}$, then there exists $P \in S L_{2}(\mathbb{C})$ such that $P \rho\left(f_{1}\right) P^{-1}$ is in the form of either (12) or (13). Since $f_{1}$ is in the center of $\pi_{1}\left(X_{1}\right)$, by Lemma $5.2 P \rho\left(\pi_{1}\left(X_{1}\right)\right) P^{-1}$ (including $P \rho\left(f_{2}\right) P^{-1}$ ) is contained in an abelian subgroup $A$ of $S L_{2}(\mathbb{C})$ ( $A$ is either the set of diagonal matrices or the set of upper triangular trace \pm 2 matrices). Since $f_{2}$ is in the center of $\pi_{1}\left(X_{2}\right)$, again by Lemma 5.2 $P \rho\left(\pi_{1}\left(X_{2}\right)\right) P^{-1}$ is also contained in $A$. Hence $\rho\left(\pi_{1}(W)\right)$, being generated by $\rho\left(\pi_{1}\left(X_{1}\right)\right), \rho\left(\pi_{1}\left(X_{2}\right)\right)$, is an abelian group. $\diamond$

Corollary 5.3. The fundamental group of the half-integral toroidal surgery on $J=k(l, m, 0,0)$ has no non-cyclic $S L_{2}(\mathbb{C})$ representations and has no non-cyclic $P S L_{2}(\mathbb{C})$ representations.

Proof. By the discussion preceding Lemma 4.4 the half-integral toroidal surgery, $M_{\mathrm{J}}(r)$, is a graph manifold satisfying the conditions of Lemma 5.1] Hence $M_{\mathrm{J}}(r)$ has no non-cyclic $S L_{2}(\mathbb{C})$ representations. The manifold cannot have non-cyclic $P S L_{2}(\mathbb{C})$ representations either since every $P S L_{2}(\mathbb{C})$ representation of $M_{\mathrm{J}}(r)$ lifts to a $S L_{2}(\mathbb{C})$-representation because the manifold has odd cyclic first homology. $\diamond$

Lemma 5.4. Let $M_{J}$ be the knot exterior of any given hyperbolic knot $J=k(l, m, 0,0)$. Let $X_{0}$ be any norm curve in $X\left(M_{J}\right)$ and $B_{0}$ the norm polygon determined by $X_{0}$. Then the half-integral toroidal slope $r=d / 2$ of $J$ is associated to a vertex of $B_{0}$ as described in Theorem 2.2 (3), i.e. 2/d is the slope of a vertex of $B_{0}$ in the $x y$-plane $H_{1}\left(\partial M_{J} ; \mathbb{R}\right)$. 
Proof. Suppose otherwise that $r$ is not associated to a vertex of $B_{0}$. As the meridian slope of $M_{\mathrm{J}}$ is not a boundary slope by Lemma 4.5 it follows from Theorem 2.2 (4) and (5) that $\mu$ is contained in $\partial B_{0}$ but $r$ is not, which means that $Z_{v}\left(\tilde{f}_{r}\right)>Z_{v}\left(\tilde{f}_{\mu}\right)$ for some point $v \in \tilde{X}_{0}$. As $M_{\mathrm{J}}(r)$ has no noncyclic representations by Lemma [5.3, the point $v$ cannot be a regular point of $\tilde{X}_{0}$ (by [9, Proposition 1.5.2] or [6. Proposition 4.8]). So $v$ is an ideal point of $\tilde{X}_{0}$. As $r$ is not a slope associated to any vertex of $B_{0}$, $\tilde{f}_{\alpha}(v)$ is finite for every class $\alpha$ in $H_{1}\left(\partial M_{\mathrm{J}} ; \mathbb{Z}\right)$. Now we may apply [6, Proposition 4.12] to see that $M_{\mathrm{J}}$ contains a closed essential surface $S$ such that if $S$ compresses in $M_{\mathrm{J}}(r)$ and $M_{\mathrm{J}}(\alpha)$, then $\Delta(r, \alpha) \leq 1$. By Lemma 4.4, $S$ must compress in $M_{\mathrm{J}}(r)$ and of course $S$ compresses in $M(\mu)$. But $\Delta(r, \mu)=2$. We arrive at a contradiction. $\diamond$

Lemma 5.5. Let $A_{J}(x, y)$ be the A-polynomial of any given hyperbolic knot $J=k(l, m, 0,0)$. Let $r=d / 2$ be the half-integral toroidal slope of $J$. If $\left(x_{0}, y_{0}\right)$ is a solution of the system

$$
\left\{\begin{array}{l}
A_{J}(x, y)=0 \\
x^{d} y^{2}-1=0
\end{array}\right.
$$

then $x_{0} \in\{0,1,-1\}$.

Proof. Suppose otherwise that $x_{0} \notin\{0,1,-1\}$. Then by the constructional definition of the $A$ polynomial, there is a component $X_{1}$ in $X^{*}\left(M_{\mathrm{J}}\right)$ which contributes a factor $f_{0}(x, y)$ in $A_{\mathrm{J}}(x, y)$ such that $\left(x_{0}, y_{0}\right)$ is a solution of

$$
\left\{\begin{array}{l}
f_{0}(x, y)=0 \\
x^{d} y^{2}-1=0
\end{array}\right.
$$

Let $Y_{0}$ be the Zariski closure of $\widehat{i}_{*}\left(X_{1}\right)$ in $X\left(\partial M_{\mathrm{J}}\right)$. We knew $Y_{0}$ is an irreducible curve. We may find an irreducible curve $X_{0}$ in $X_{1}$ such that $Y_{0}$ is also the Zariski closure of $\widehat{i}_{*}\left(X_{0}\right)$. Now it also follows from the constructional definition of the $A$-polynomial that there is a convergent sequence of regular points $\left\{v_{i}\right\} \subset \tilde{X}_{0}$ such that $\tilde{f}_{r}\left(v_{i}\right) \rightarrow 0$ and $\tilde{f}_{\mu}\left(v_{i}\right) \rightarrow\left(x_{0}+x_{0}^{-1}\right)^{2}-4$, i.e. if $v$ is the limit point of $v_{i}$ in $\tilde{X}_{0}$, then $\tilde{f}_{r}(v)=0$ and $\tilde{f}_{\mu}(v)=\left(x_{0}+x_{0}^{-1}\right)^{2}-4 \neq 0$.

Note that $\tilde{f}_{r}$ is not constant on $X_{0}$. For otherwise $X_{0}$ would be a semi-norm curve with $r$ as the associated slope and would have a contradiction with Theorem 2.5 (3) (since by Lemma $4.5 \mu$ is not a boundary slope).

So we have $Z_{v}\left(\tilde{f}_{r}\right)>Z_{v}\left(\tilde{f}_{\mu}\right)=0$. Again $v$ cannot be a regular point of $\tilde{X}_{0}$ due to Lemma 5.3 So $v$ is an ideal point of $\tilde{X}_{0}$ such that $f_{r}(v)=0$ and $f_{\mu}(v) \neq 0$ is finite. We get a contradiction with [6, Prposition 4.12] as in the proof of Lemma [5.4 $\diamond$

Lemma 5.6. Let $K$ be a hyperbolic knot in $S^{3}$. For a given slope $p / q$, if every solution $\left(x_{0}, y_{0}\right)$ of the system of equations

$$
\left\{\begin{array}{l}
A_{K}(x, y)=0 \\
x^{p} y^{q}-1=0
\end{array}\right.
$$

has $x_{0} \in\{1,-1,0\}$, then $M_{K}(p / q)$ is not a hyperbolic manifold.

Proof. Some of the ideas for the proof come from [5]. Suppose otherwise that $M_{\mathrm{K}}(p / q)$ is a hyperbolic 3-manifold. Then $\pi_{1}\left(M_{\mathrm{K}}(p / q)\right)$ has a discrete faithful representation $\bar{\rho}_{0}$ into $P S L_{2}(\mathbb{C})$. By Thurston (10, Proposition 3.1.1]), this representation can be lifted to a $S L_{2}(\mathbb{C})$-representation $\rho_{0}$. It follows from the Mostow rigidity that the character $\chi_{\rho_{0}}$ of $\rho_{0}$ is an isolated point in $X\left(M_{\mathrm{K}}(p / q)\right)$. Note that $\rho_{0}$ can be considered as an element in $R\left(M_{\mathrm{K}}\right)$ and $\chi_{\rho_{0}}$ can be considered as an element in $X\left(M_{\mathrm{K}}\right)$ since $R\left(M_{\mathrm{K}}(p / q)\right)$ embeds in $R\left(M_{\mathrm{K}}\right)$ and $X\left(M_{\mathrm{K}}(p / q)\right)$ embeds in $X\left(M_{\mathrm{K}}\right)$. Of course we have $\rho_{0}\left(\mu^{p} \lambda^{q}\right)=I$ but $\rho_{0}(\mu) \neq \pm I$. Let $X_{0}$ be a component of $X\left(M_{\mathrm{K}}\right)$ which contains $\chi_{\rho_{0}}$. By Thurston ([10, Proposition 3.2.1]), $X_{0}$ is positive dimensional.

Claim 5.7. The function $f_{\mu^{p} \lambda^{q}}$ is non-constant on $X_{0}$.

Suppose otherwise. Then $f_{\mu^{p} \lambda^{q}}$ is constantly zero on $X_{0}$ since $f_{\mu^{p} \lambda^{q}}\left(\chi_{\rho_{0}}\right)=0$. So for every $\chi_{\rho} \in X_{0}$, $\rho\left(\mu^{p} \lambda^{q}\right)$ is either $I$ or $-I$ or is a parabolic element. Let $X_{1}$ be an irreducible curve in $X_{0}$ which contains 
the point $\chi_{\rho_{0}}$. For a generic point $\chi_{\rho} \in X_{1}, \rho\left(\mu^{p} \lambda^{q}\right)$ cannot be a parabolic element since otherwise $\rho(\mu)$ is either $I$ or $-I$ or parabolic for all $\chi_{\rho} \in X_{1}$ and this happens in particular at the point $\chi_{\rho_{0}}$, yielding a contradiction. For a generic point $\chi_{\rho} \in X_{1}, \rho\left(\mu^{p} \lambda^{q}\right)$ cannot be $-I$ either for otherwise by continuity, $\rho\left(\mu^{p} \lambda^{q}\right)=-I$ for every point $\chi_{\rho} \in X_{1}$, and this happens in particular at the point $\chi_{\rho_{0}}$, yielding another contradiction. So for a generic point $\chi_{\rho} \in X_{1}, \rho\left(\mu^{p} \lambda^{q}\right)=I$ and again by continuity, $\rho\left(\mu^{p} \lambda^{q}\right)=I$ for every point $\chi_{\rho} \in X_{1}$. So $X_{1}$ factors though the $p / q$-surgery on $K$ and becomes a subvariety of $X\left(M_{\mathrm{K}}(p / q)\right)$. But this contradicts the fact that $\chi_{\rho_{0}}$ is an isolated point of $X\left(M_{\mathrm{K}}(p / q)\right)$. The claim is thus proved.

It also follows from the proof of Claim 5.7 that $X_{0}$ is one dimensional. For otherwise there would be a curve $X_{1}$ in $X_{0}$ such that $\chi_{\rho_{0}} \in X_{1}$ and $f_{\mu^{p} \lambda^{q}}$ is constantly zero on $X_{1}$, which is impossible by the proof of Claim 5.7

It follows from Claim 5.7that the restriction of $X_{0}$ in $X\left(\partial M_{\mathrm{K}}\right)$ is one dimensional and thus $X_{0} \in X^{*}\left(M_{\mathrm{K}}\right)$ and contributes a factor $f_{0}(x, y)$ to $A_{\mathrm{K}}(x, y)$.

We may assume, up to conjugation of $\rho_{0}$, that

$$
\rho_{0}(\mu)=\left(\begin{array}{cc}
x_{0} & a \\
0 & x_{0}^{-1}
\end{array}\right), \quad \rho_{0}(\lambda)=\left(\begin{array}{cc}
y_{0} & b \\
0 & y_{0}^{-1}
\end{array}\right) .
$$

Note that $x_{0} \neq \pm 1\left(\right.$ as $\rho_{0}(\mu) \neq \pm I$ and cannot be a parabolic element of $\left.S L_{2}(\mathbb{C})\right)$. By the construction of $A_{\mathrm{K}}(x, y),\left(x_{0}, y_{0}\right)$ is a solution of the system

$$
\left\{\begin{array}{l}
f_{0}(x, y)=0 \\
x^{p} y^{q}-1=0 .
\end{array}\right.
$$

and thus is a solution of

$$
\left\{\begin{array}{l}
A_{\mathrm{K}}(x, y)=0 \\
x^{p} y^{q}-1=0 .
\end{array}\right.
$$

We get a contradiction with the assumption of the lemma.

Proposition 5.8. If $K \subset S^{3}$ is a hyperbolic knot whose A-polynomial divides the A-polynomial of $J=k(l, m, 0,0)$, then $K$ has the same half-integral toroidal slope as $J$ and thus $K$ is one of $k(l, m, n, p)$.

Proof. Since $K$ is hyperbolic, $X\left(M_{\mathrm{K}}\right)$ contains a norm curve component $X_{0}^{\prime}$ which contributes a balanced-irreducible factor $f_{0}(x, y)$ to $A_{\mathrm{K}}(x, y)$ such that the Newton polygon of $f_{0}(x, y)$ is dual to the norm polygon determined by $X_{0}^{\prime}$ by Theorem 2.3 By the assumption that $A_{\mathrm{K}}(x, y)$ divides $A_{\mathrm{J}}(x, y)$, $f_{0}(x, y)$ is also a factor of $A_{\mathrm{J}}(x, y)$. Thus there is a curve $X_{0}$ in a component of $X^{*}\left(M_{\mathrm{J}}\right)$ which contributes $f_{0}(x, y)$ and $X_{0}$ must be a norm curve whose norm polygon $B_{0}$ is dual to the Newton polygon of $f_{0}(x, y)$. By Lemma [5.4 the half-integral toroidal slope $r=d / 2$ of $J$ is associated to a vertex of $B_{0}$ and thus $r=d / 2$ is also associated to an edge of the Newton polygon of $f_{0}(x, y)$. Hence $r$ is also a boundary slope of $K$.

Again by the assumption that $A_{\mathrm{K}}(x, y)$ divides $A_{\mathrm{J}}(x, y)$, together with Lemma 5.5. we see that if $\left(x_{0}, y_{0}\right)$ is a solution of the system

$$
\left\{\begin{array}{l}
A_{\mathrm{K}}(x, y)=0 \\
x^{d} y^{2}-1=0
\end{array}\right.
$$

then $x_{0} \in\{0,1,-1\}$. Now applying Lemma [5.6] we see that $M_{\mathrm{K}}(r)$ is not a hyperbolic manifold. Applying [9, Theorem 2.0.3] and [16] we see that $M_{\mathrm{K}}(r)$ must be a Haken manifold and thus must be a toroidal manifold (as it has finite first homology). Finally $K$ is one of the $\operatorname{knots} k(l, m, n, p)$ by Theorem 4.1 $\diamond$

Lemma 5.9. The half-integral toroidal $r$-surgery on $J=k(l, m, n, 0), n \neq 0,1$, is a manifold with an irreducible $S L_{2}(\mathbb{C})$ representation $\rho_{0}$ whose image contains no parabolic elements.

Proof. We knew from Section 4 that $M_{\mathrm{J}}(r)=X_{1} \cup X_{2}$, where $X_{1}$ is a $(a, b)$-torus knot exterior and $X_{2}$ is Seifert fibred with base orbifold $D^{2}(2, c)$ for some odd integer $c>1$, such that the meridian slope $\mu_{1}$ 
of $X_{1}$ is identified with the Seifert fibre slope $\sigma_{2}$ of $X_{2}$ and the Seifert fiber slope $\sigma_{1}$ of $X_{1}$ is identified with a lens space filling slope $\mu_{2}$ of $X_{2}$ (and the lens space has order $\left.|2 n-1|\right)$.

Perhaps it is easier to construct a $P S L_{2}(\mathbb{C})$ representation $\bar{\rho}_{0}$ of $\pi_{1}\left(M_{\mathrm{J}}(r)\right)$ with the required properties. As $M_{\mathrm{J}}(r)$ has zero $\mathbb{Z}_{2}$-homology, every $P S L_{2}(\mathbb{C})$ representation of $\pi_{1}\left(M_{\mathrm{J}}(r)\right)$ lifts to a $S L_{2}(\mathbb{C})$ representation.

The representation $\bar{\rho}_{0}$ will send $\pi_{1}\left(X_{2}\right)$ to a cyclic group of order $|2 n-1|$ which is possible by factoring through $X_{2}\left(\mu_{2}\right)$. So $\bar{\rho}_{0}\left(\mu_{2}\right)=i d$. We claim $\bar{\rho}_{0}\left(\sigma_{2}\right)$ is not the identity element. For otherwise $\bar{\rho}_{0}$ factors through the group

$$
<x, y ; x^{2}=y^{c}=1, x y=1>
$$

which is the trivial group as $c$ is odd.

Hence the order of $\bar{\rho}_{0}\left(\sigma_{2}\right)$ is an odd number $q>1$ which is a factor of $2 n-1$.

On the $X_{1}$ side, we need to have $\bar{\rho}_{0}\left(\sigma_{1}\right)=i d$ and $\bar{\rho}_{0}\left(\mu_{1}\right)=\bar{\rho}_{0}\left(\sigma_{2}\right)$ of order $q$. So $\bar{\rho}_{0}$ factors through the triangle group

$$
<x, y ; x^{a}=y^{b}=(x y)^{q}=1>.
$$

Such representation exists and can be required to be irreducible. Also as at least one of $a$ and $b$ is odd and $q$ is odd, we may require the image of $\bar{\rho}_{0}$ containing no parabolic elements. In fact the triangle group is either a spherical or a hyperbolic triangle group and so we may simply choose $\bar{\rho}_{0}$ to be a discrete faithful representation of the triangle group into $S O(3) \subset P S L_{2}(\mathbb{C})$ (when the triangle group is spherical) or into $P S L_{2}(\mathbb{R}) \subset P S L_{2}(\mathbb{C})$ (when the triangle group is hyperbolic), and thus the image of $\bar{\rho}_{0}$ has no parabolic elements. $\diamond$

Lemma 5.10. For any given $J=k(l, m, 0, p)$, with $p \neq 0,1$, and with $l$ not divisible by $|2 p-1|$, the half-integral toroidal surgery on $J$ is a manifold with an irreducible $S L_{2}(\mathbb{C})$ representation $\rho_{0}$ whose image contains no parabolic elements.

Proof. The proof is similar to that of Lemma 5.9.

We knew $M_{\mathrm{J}}(r)=X_{1} \cup X_{2}$, where $X_{2}$ is a $(2, a)$-torus knot exterior and $X_{1}$ is Seifert fibred with base orbifold $D^{2}(|l|,|-\operatorname{lm}(2 p-1)+p l+2 p-1|)$, such that the meridian slope $\mu_{2}$ of $X_{2}$ is identified with the Seifert fibre slope $\sigma_{1}$ of $X_{1}$ and the Seifert fiber slope $\sigma_{2}$ of $X_{2}$ is identified with a lens space filling slope $\mu_{1}$ of $X_{1}$ (and the lens space has order $|2 p-1|$ ).

As in Lemma [5.9] we jus need to construct a $P S L_{2}(\mathbb{C})$ representation $\bar{\rho}_{0}$ of $\pi_{1}\left(M_{\mathrm{J}}(r)\right)$ which is irreducible and whose image contains no parabolic elements.

The representation $\bar{\rho}_{0}$ will send $\pi_{1}\left(X_{1}\right)$ to a cyclic group of order $|2 p-1|$ which is possible by factoring through $X_{1}\left(\mu_{1}\right)$. So $\bar{\rho}_{0}\left(\mu_{1}\right)=i d$. We claim $\bar{\rho}_{0}\left(\sigma_{1}\right)$ is not the identity element. For otherwise $\bar{\rho}_{0}$ factors through the group

$$
<x, y ; x^{l}=y^{-l m(2 p-1)+p l+2 p-1}=1, x y=1>
$$

which is a cyclic group of order less than $|2 p-1|$ since $l$ is not divisible by $2 p-1$ by our assumption.

Hence the order of $\bar{\rho}_{0}\left(\sigma_{2}\right)$ is an odd number $q>1$ which is a factor of $2 p-1$.

On the $X_{2}$ side, we need to have $\bar{\rho}_{0}\left(\sigma_{2}\right)=i d$ and $\bar{\rho}_{0}\left(\mu_{2}\right)=\bar{\rho}_{0}\left(\sigma_{1}\right)$ of order $q$. So $\bar{\rho}_{0}$ factors through the triangle group

$$
<x, y ; x^{2}=y^{a}=(x y)^{q}=1>.
$$

Such representation exists and can be required to be irreducible. Also as both $a$ and $q$ are odd, we may require the image of $\bar{\rho}_{0}$ containing no parabolic elements.

Lemma 5.11. The A-polynomial of any $J=k(l, m, n, 0), n \neq 0,1$, does not divide the A-polynomial of any $J^{\prime}=k\left(l^{\prime}, m^{\prime}, 0,0\right)$.

Proof. Suppose otherwise that $A_{\mathrm{J}}(x, y) \mid A_{\mathrm{J}^{\prime}}(x, y)$. Then by Proposition $5.8 J$ and $J^{\prime}$ have the same half-integral toroidal slope $r=d / 2, d$ odd. 
Let $\rho_{0}$ be an irreducible representation of $M_{\mathrm{J}}(r)$ provided by Lemma 5.9. Then $\rho_{0}(r)=I$ but $\rho_{0}(\mu) \neq \pm I$. We know that $\chi_{\rho_{0}}$ is contained in a positive dimensional component $X_{1}$ of $X\left(M_{\mathrm{J}}\right)$. Let $X_{0}$ be an irreducible curve in $X_{1}$ containing $\chi_{\rho_{0}}$.

Claim. $f_{r}$ is not constant on $X_{0}$.

Otherwise $f_{r}$ is constantly equal to 4 on $X_{0}$. If $f_{\mu}$ is not a constant on $X_{0}$, then $X_{0}$ would be a semi-norm with $r$ as its associated boundary slope, which is impossible by Theorem 2.5 as $\mu$ is not a boundary slope and $\Delta(\mu, r)=2$. So $f_{\mu}$ is a constant not equal to 4 on $X_{0}$ since $\rho_{0}(\mu) \neq \pm I$ and is not parabolic. So for any point $\chi_{\rho} \in X_{0}, \rho(r)$ cannot be a parabolic element (for otherwise $\rho(\mu)$ is also parabolic and thus $\left.f_{\mu}\left(\chi_{\rho}\right)=4\right)$. So $\rho(r)=I$ for any $\chi_{\rho} \in X_{0}$. We now get a contradiction with [6, Proposition 4.10], which proves the claim.

So $f_{r}$ is not constant on $X_{0}$ which means that the component $X_{1} \supset X_{0}$ belongs to $X^{*}\left(M_{\mathrm{J}}\right)$ and thus contributes a factor in $A_{\mathrm{J}}(x, y)$. Moreover the point $\chi_{\rho_{0}}$ contributes a root $\left(x_{0}, y_{0}\right)$ to the system

$$
\left\{\begin{array}{l}
A_{\mathrm{J}}(x, y)=0 \\
x^{d} y^{2}=1
\end{array}\right.
$$

such that $x_{0} \neq \pm 1,0$. As $A_{\mathrm{J}}(x, y) \mid A_{\mathrm{J}^{\prime}}(x, y),\left(x_{0}, y_{0}\right)$ is also a solution of the system

$$
\left\{\begin{array}{l}
A_{J^{\prime}}(x, y)=0 \\
x^{d} y^{2}=1
\end{array}\right.
$$

which contradicts Lemma [5.5. $\diamond$

With a similar proof replacing Lemma 5.9 by Lemma 5.10 , we have

Lemma 5.12. The A-polynomial of any $k(l, m, 0, p), p \neq 0,1, l$ not divisible by $2 p-1$, does not divide the A-polynomial of any $k\left(l^{\prime}, m^{\prime}, 0,0\right)$.

Lemma 5.13. Let $K$ be a hyperbolic knot in $S^{3}$. For a given slope $p / q, p$ odd, if every solution $\left(x_{0}, y_{0}\right)$ of the system of equations

$$
\left\{\begin{array}{l}
A_{K}(x, y)=0 \\
x^{p} y^{q}-1=0
\end{array}\right.
$$

has $x_{0} \in\{1,-1,0\}$, then $M_{K}(p / q)$ cannot be a Seifert fibred space whose base orbifold is a 2 -sphere with exactly three cone points.

Proof. The proof is similar to that of Lemma 5.6. Suppose otherwise that $M_{\mathrm{K}}(p / q)$ is a a Seifert fibred space whose base orbifold is a 2-sphere with exactly three cone points. As $p$ is odd, the base orbifold is either spherical or hyperbolic. Hence $\pi_{1}\left(M_{\mathrm{K}}(p / q)\right)$ has an irreducible $P S L_{2}(\mathbb{C})$ representation $\bar{\rho}_{0}$ which factors through the orbifold fundamental group of the base orbifold such that the image of $\bar{\rho}_{0}$ contains no parabolic elements. As $p$ is odd, the $P S L_{2}(\mathbb{C})$ representation $\bar{\rho}_{0}$ lifts to a $S L_{2}(\mathbb{C})$ representation $\rho_{0}$. It is well known that the character $\chi_{\rho_{0}}$ of $\rho_{0}$ is an isolated point in $X\left(M_{\mathrm{K}}(p / q)\right)$. Considered as a point in $X\left(M_{\mathrm{K}}\right), \chi_{\rho_{0}}$ is contained in a positive dimensional component $X_{0}$ of $X\left(M_{\mathrm{K}}\right)$. Arguing exactly as in Claim 5.7 we have $f_{\mu^{p} \lambda^{q}}$ is non-constant on $X_{0}$. Hence $X_{0}$ is contained $X^{*}\left(M_{\mathrm{K}}\right)$ and contributes a factor $f_{0}(x, y)$ to $A_{\mathrm{K}}(x, y)$. Exactly as in the proof of Lemma 5.6. the point $\chi_{\rho_{0}}$ provides a solution $\left(x_{0}, y_{0}\right)$ to the system

$$
\left\{\begin{array}{l}
A_{\mathrm{K}}(x, y)=0 \\
x^{p} y^{q}-1=0
\end{array}\right.
$$

such that $x_{0} \notin\{1,-1,0\}$, giving a contradiction with the assumption of the lemma. $\diamond$

\section{Distinguishing $k\left(l_{*},-1,0,0\right)$ from $k(l, m, 0, p)$}

The goal of this section is to prove the following proposition:

Proposition 6.1. Suppose that two knots $k\left(l_{*},-1,0,0\right)$ and $k(l, m, 0, p)$ have the same genus $g$ and the same half-integral toroidal slope $r$, where $l_{*}>1$ and $(1-2 p) \mid l$, then $k\left(l_{*},-1,0,0\right)=k(l, m, 0, p)$. 
Let

$$
s=r+\frac{1}{2}, \quad d=-s-2 g
$$

For $k(l, m, 0, p)$, by (9),

$$
-s=-p(2 m l-l-1)^{2}+(2 m l-l)(m l-1) .
$$

When $p \leq 0$, using (10), we get

$$
d= \begin{cases}-p(2 m l-l-1)+3 m l-l-2 \alpha, & \text { if } l m>0, \\ -p(-2 m l+l+1)-3 m l+l, & \text { if } l m<0,\end{cases}
$$

where

$$
\alpha= \begin{cases}1, & \text { if } l>0, m>0 \\ 2, & \text { if } l<0, m<0 .\end{cases}
$$

Consider the family of knots $k\left(l_{*},-1,0,0\right)$, where $l_{*}>1$. In this case, $d, s$ are given by

$$
\left\{\begin{aligned}
d & =4 l_{*} \\
s & =-3 l_{*}\left(l_{*}+1\right)
\end{aligned}\right.
$$

so

$$
-\frac{4}{3} s+1=\left(\frac{d}{2}+1\right)^{2}
$$

Lemma 6.2. Suppose that $p<0$ and $(1-2 p) \mid l$, then the knot $k(l, m, 0, p)$ has different $(g, r)$ from the knot $k\left(l_{*},-1,0,0\right)$, where $l_{*}>1$.

Proof. Otherwise, assume $k(l, m, 0, p)$ has the same $(g, r)$ as $k\left(l_{*},-1,0,0\right)$. Using (15), we see that

$$
d+2>-p|2 m l-l-1|+|2 m l-l-1| .
$$

Using (14) and (8), we have

$$
-s+\frac{3}{4}<-p(2 m l-l-1)^{2}+(2 m l-l)(m l-1)+1<(1-p)(2 m l-l-1)^{2} .
$$

Using (17) and the previous two inequalities, we get

$$
\begin{aligned}
\frac{(1-p)^{2}}{4}(2 m l-l-1)^{2} & <\left(\frac{d}{2}+1\right)^{2} \\
& =-\frac{4}{3} s+1 \\
& <\frac{4}{3}(1-p)(2 m l-l-1)^{2},
\end{aligned}
$$

so $1-p<\frac{16}{3}$, hence $-p \leq 4$.

If $p=-1$ or $-4,3 \mid l$. By (14), $3 \nmid-s$, which contradicts (16).

If $p=-3$, then $7 \mid l$. By (14),$-s \equiv 3(\bmod 7)$. It follows from (17) that 5 is a quadratic residue modulo 7 , which is not true.

If $p=-2$, then $5 \mid l$ and $-s \equiv 2(\bmod 5)$. It follows from (17) that 2 is a quadratic residue modulo 5 , which is not true. $\diamond$ 
For the family of knots $k(l, m, 0,0)$, the following proposition expresses $l, m$ in terms of $s$ and $d$.

Proposition 6.3. For the family of knots $k(l, m, 0,0)$, the pair $(l, m)$ is determined by $s$ and $d$ by the following formulas:

If $l m>0$, then

$$
\begin{aligned}
l & =\frac{d+2 \alpha+3-3 \sqrt{(d+2 \alpha-1)^{2}+4 s}}{2} \\
m & =\frac{1}{3}\left(\frac{2 d+4 \alpha}{d+2 \alpha+3-3 \sqrt{(d+2 \alpha-1)^{2}+4 s}}+1\right) .
\end{aligned}
$$

If $\operatorname{lm}<0$, then

$$
\begin{aligned}
l & =\frac{-d+3+3 \sqrt{(d+1)^{2}+4 s}}{2} \\
m & =\frac{1}{3}\left(1-\frac{2 d}{-d+3+3 \sqrt{(d+1)^{2}+4 s}}\right) .
\end{aligned}
$$

Proof. Using (15), we can express $m l$ as a linear function of $d$ and $l$. Substitute such expression of $m l$ into (14), we get

$$
-s= \begin{cases}\frac{1}{9}(-l+2 d+4 \alpha)(l+d+2 \alpha-3), & \text { if } l m>0, \\ \frac{1}{9}(-l-2 d)(l-d-3), & \text { if } l m<0 .\end{cases}
$$

If $l m>0$, by (18), $l$ is a root of the quadratic polynomial

$$
(x-2 d-4 \alpha)(x+d+2 \alpha-3)-9 s,
$$

whose two roots are

$$
\frac{d+2 \alpha+3 \pm 3 \sqrt{(d+2 \alpha-1)^{2}+4 s}}{2} .
$$

By (15), $d>0$ and $|2 l| \leq d+2 \alpha$, so

$$
l=\frac{d+2 \alpha+3-3 \sqrt{(d+2 \alpha-1)^{2}+4 s}}{2} .
$$

Using (15) again, we can compute $m$ as in the statement.

If $l m<0, l$ is a root of the quadratic polynomial

$$
(x+2 d)(x-d-3)-9 s,
$$

whose two roots are

$$
\frac{-d+3 \pm 3 \sqrt{(d+1)^{2}+4 s}}{2} .
$$

By (15), $d>0$ and $|2 l| \leq d$, so

$$
l=\frac{-d+3+3 \sqrt{(d+1)^{2}+4 s}}{2} .
$$

Using (15) again, we can compute $m$ as in the statement. $\diamond$ 
Lemma 6.4. Suppose that two knots $k(l, m, 0,0)$ and $k\left(l_{*}, m_{*}, 0,0\right)$ have the same $g$ and $r$. Suppose further that

$$
l m, l_{*} m_{*}>0, \quad l>0>l_{*} .
$$

Then the quadruple $\left(l, m, l_{*}, m_{*}\right)$ is either $(2,2,-3,-1)$ or $(6, m,-2,-3 m+1)$ for $m \geq 2$.

Proof. Using Proposition 6.3 and (8), we get

$$
l=\frac{d+5-3 \sqrt{(d+1)^{2}+4 s}}{2} \geq 2
$$

and

$$
l_{*}=\frac{d+7-3 \sqrt{(d+3)^{2}+4 s}}{2} \leq-2
$$

Hence

which implies

$$
\frac{(d+11)^{2}}{9}-(d+3)^{2} \leq 4 s \leq-\frac{8}{9}(d+1)^{2}
$$

$$
\frac{1}{9}(d-7)^{2} \leq(d+1)^{2}+4 s \leq \frac{1}{9}(d+1)^{2} .
$$

From (15) and (8), we can conclude that

$$
d \geq 8
$$

By (21), $(d+1)^{2}+4 s$ is a perfect square which has the same parity as $d+1$, hence

$$
(d+1)^{2}+4 s=\frac{(d+1-2 c)^{2}}{9}
$$

for some $c \in\{0,1,2,3,4\}$. Then

$$
l=c+2, \quad m=\frac{d+c+4}{3(c+2)} .
$$

Using (23), we also get

$$
\frac{(d+11)^{2}}{9} \leq(d+3)^{2}+4 s \leq \frac{d^{2}+38 d+73}{9}<\frac{(d+19)^{2}}{9} .
$$

By (22), $(d+3)^{2}+4 s$ is a perfect square with the same parity as $d+3$, so

$$
(d+3)^{2}+4 s=\frac{\left(d+11+2 c_{*}\right)^{2}}{9}
$$

for some $c_{*} \in\{0,1,2,3\}$. Then

$$
l_{*}=-c_{*}-2, \quad m_{*}=-\frac{d-c_{*}+2}{3\left(c_{*}+2\right)} .
$$

Compare (25) and (26), we get

$$
\left(4-c_{*}-c\right) d=\left(5+c_{*}+c\right)\left(6+c_{*}-c\right)-18 .
$$

Moreover, since both $\frac{d+1-2 c}{3}$ and $\frac{d+11+2 c_{*}}{3}$ are integers, $\frac{10+2 c_{*}+2 c}{3}$ is an integer, so

$$
c+c_{*} \in\{1,4,7\} \text {. }
$$

If $c+c_{*}=7$, then $c=4, c_{*}=3$. By (27), $d=-14$, a contradiction to (24).

If $c+c_{*}=4$, using (27) we get $c=4, c_{*}=0$. Hence $l=6, m=\frac{d+8}{18}, l_{*}=-2, m_{*}=-\frac{d+2}{6}=-3 m+1$.

If $c+c_{*}=1$, using (27) we get $d=6+2\left(c_{*}-c\right)$. Using (24), the only possible case is $c=0, c_{*}=1$, and $\left(l, m, l_{*}, m_{*}\right)=(2,2,-3,-1) . \diamond$ 
Proof of Proposition 6.1. When $p<0$, this result follows from Lemma 6.2.

When $p>0$, from the formula (14) it is easy to see $s>0$ for $k(l, m, 0, p)$, but $s=-3 l_{*}\left(l_{*}+1\right)<0$ for $k\left(l_{*},-1,0,0\right)$.

Now we consider the case $p=0$. By Proposition 6.3 for any given $(g, r)$, there are at most three knots $k\left(l_{i}, m_{i}, 0,0\right), i=1,2,3$, having this $(g, r)$. There is at most one pair $\left(l_{i}, m_{i}\right)$ in each of the three cases:

- $l>0, m>0$,

- $l<0, m<0$,

- $l m<0$.

The pair $\left(l_{*},-1\right)$ is in the third case. By Proposition 4.2 (c), $k\left(-l_{*}-1,-1,0,0\right)=k\left(l_{*},-1,0,0\right)$, and $\left(-l_{*}-1,-1\right)$ is in the second case. Suppose that there is also a pair $(l, m)$ in the first case with the same $(g, r)$, then Lemma 6.4 implies that $(l, m)=(2,2)$ and $-l_{*}-1=-3$. By Proposition $4.2(\mathrm{~d})$, the three knots $k(2,2,0,0), k(-3,-1,0,0), k(2,-1,0,0)$ are the same. $\diamond$

\section{Proof of Theorem 1.2}

Recall that the unique half-integral toroidal slope of $k\left(l_{*},-1,0,0\right)$ is $r=(2 s-1) / 2$, where $s=-3 l_{*}\left(l_{*}+1\right)$. Hence the family of knots $\left\{k\left(l_{*},-1,0,0\right) ; l_{*}>1\right\}$ are mutually distinct.

Suppose that $K \subset S^{3}$ is a knot which has the same $A$-polynomial and the same knot Floer homology as a given $J_{*}=k\left(l_{*},-1,0,0\right), l_{*}>1$. Our goal is to show that $K=J_{*}$.

As $J_{*}$ is hyperbolic, $K$ cannot be a torus knot by Theorem 1.1.

Suppose $K$ is hyperbolic. Then by Lemma 5.8, $K$ has the same half-integral toroidal slope $r$ as $J_{*}$ and $K$ is one of $k(l, m, n, p)$. Applying Lemma [5.11, we have $n=0$ or 1 . Since the half-integral toroidal slope of $k(l, m, 1,0)$ is positive while the half-integral toroidal slope of $J_{*}=k\left(l_{*},-1,0,0\right)$ is negative, we see that $n$ must be zero. Similarly applying Lemma [5.12, we have $p$ is non-positive, and $2 p-1$ divides $l$. That is, we have $K=k(l, m, 0, p)$ with $(2 p-1) \mid l$. Now by Proposition 6.1, we have $K=J_{*}$.

It remains to show that $K$ cannot be a satellite knot. Suppose otherwise that $K$ is a satellite knot. We are going to derive a contradiction from this assumption.

Lemma 7.1. The A-polynomial $A_{J_{*}}(x, y)$ of $J_{*}=k\left(l_{*},-1,0,0\right)$ does not contain any factor of the form $x^{j} y+\delta$ or $y+\delta x^{-j}$ for $j \in \mathbb{Z}$ and $\delta \in\{-1,1\}$.

Proof. Suppose otherwise that $A_{\mathrm{J}_{*}}(x, y)$ contains a factor of the form $x^{j} y+\delta$ or $y+\delta x^{-j}$. As this factor is irreducible and balanced, it is contributed by a curve component $X_{0}$ in $X^{*}\left(M_{\mathrm{J}_{*}}\right)$. Moreover $X_{0}$ is a semi-norm curve with $\mu^{j} \lambda$ as the unique associated boundary slope.

We claim that $\mu^{j} \lambda$ is either $\mu^{s} \lambda$ or $\mu^{s-1} \lambda$. From Theorem 2.5 (3) and Lemma4.5, we see that the meridian slope $\mu$ has the minimal semi-norm $s_{0}$. To prove the claim, we just need to show, by Theorem 2.5 (4), that for the half-integral toroidal slope $r$ of $J_{*}$, we have $\|r\|_{0}=\|\mu\|_{0}$, which is equivalent to show that

$$
Z_{v}\left(\tilde{f}_{r}\right) \leq Z_{v}\left(\tilde{f}_{\mu}\right)
$$

for every $v \in \tilde{X}_{0}$. As $M_{\mathrm{J}_{*}}(r)$ has no non-cyclic representation by Lemma 5.3, at every regular point $v \in \tilde{X}_{0}$ we have $Z_{v}\left(\tilde{f}_{r}\right) \leq Z_{v}\left(\tilde{f}_{\mu}\right)$. If at an ideal point $v$ of $\tilde{X}_{0}, Z_{v}\left(\tilde{f}_{r}\right)>Z_{v}\left(\tilde{f}_{\mu}\right)$, then $\tilde{f}_{\alpha}(v)$ is finite for every class $\alpha$ in $H_{1}\left(\partial M_{J_{*}} ; \mathbb{Z}\right)$ (because both $\tilde{f}_{r}$ and $\tilde{f}_{\mu^{j} \lambda}$ are finite at $\left.v\right)$. We can now derive a contradiction with [6. Proposition 4.12] just as in the proof of Lemma 5.4 and the claim is thus proved.

On the other hand it is shown in [13, Proposition 5.4] that each of $M_{\mathrm{J}_{*}}(s)$ and $M_{\mathrm{J}_{*}}(s-1)$ is a small Seifert fibred space. As $J_{*}$ is a small knot, each of $\mu^{s} \lambda$ and $\mu^{s-1} \lambda$ cannot be a boundary slope by 9 , Theorem 2.0.3] and thus cannot be the slope $\mu^{j} \lambda$. We arrive at a contradiction. $\diamond$ 
Since $J_{*}$ is a fibered knot by [13, $K$ is also fibered. Hence if $(C, P)$ is any pair of companion knot and pattern knot associated to $K$, then each of $C$ and $P$ is fibred and the winding number $w$ of $P$ with respect to $C$ is larger than zero.

Lemma 7.2. The satellite knot $K$ has a companion knot $C$ which is hyperbolic.

Proof. As is true for any satellite knot, $K$ has a companion knot $C$ which is either a torus knot or a hyperbolic knot. So we just need to rule out the possibility that $C$ be a torus knot. If $C$ is a $(p, q)$-torus knot, then by formula (3),$A_{\mathrm{C}}(x, y)$ contains a factor of the form $y x^{k}+\delta$ or $y+\delta x^{-k}$ for some integer $k$ and $\delta \in\{1,-1\}$. As the winding number $w$ of the pattern knot $P$ with respect to $C$ is non-zero, by Proposition 2.8, $A_{\mathrm{K}}(x, y)$ contains a factor of the form $y x^{w^{2} k}-(-\delta)^{w}$ or $y-(-\delta)^{w} x^{-w^{2} k}$. But this contradicts Lemma $7.1 \diamond$

We now fix a hyperbolic companion knot $C$ for $K$ which exists by Lemma 7.2 and let $P$ be the corresponding pattern knot.

Lemma 7.3. For any hyperbolic knot $C$ in $S^{3}$, any surgery with a slope $j / k, \operatorname{gcd}(j, k)=1$, satisfying $k>2$ and $j$ odd, will produce either a hyperbolic manifold or a Seifert fibred space whose base orbifold is $S^{2}$ with exactly three cone points.

Proof. As $k>2, M_{\mathrm{C}}(j / k)$ is irreducible [16] and atoroidal [17. Thus $M_{\mathrm{C}}(j / k)$ is either a hyperbolic manifold or an atoroidal Seifert fibred space. In latter case, the Seifert fibered space has non-cyclic fundamental group [9]. As $p$ is odd, the base orbifold of the Seifert fibered space cannot be nonorientable. Thus the base orbifold is a 2 -sphere with exactly three cone points. $\diamond$

Lemma 7.4. The integer $d=2 s-1$ is divisible by $w^{2}$.

Proof. Suppose otherwise. Let $d_{1} / q_{1}$ be the rational number $\frac{d}{2 w^{2}}$ in its reduced form, i.e. $d_{1}=$ $d / \operatorname{gcd}\left(d, w^{2}\right)$ and $q_{1}=2 w^{2} / \operatorname{gcd}\left(d, w^{2}\right)$. Then $q_{1}>2$ and $d_{1}$ is odd. So by Lemma 7.3, the surgery on $C$ with the slope $d_{1} / q_{1}$ will yield either a hyperbolic manifold or a Seifert fibred space whose base orbifold is a 2-sphere with exactly three cone points. Applying either Lemma 5.6 or Lemma 5.13 we see that the $A$-polynomial $A_{\mathrm{C}}(\bar{x}, \bar{y})$ of $C$ has a zero point $\left(\bar{x}_{0}, \bar{y}_{0}\right)$ such that $\bar{x}_{0}^{d_{1}} \bar{y}_{0}^{q_{1}}=1$ and $\bar{x}_{0} \notin\{0,1,-1\}$. Now from Proposition 2.8 and its proof, we see that $A_{\mathrm{C}}(\bar{x}, \bar{y})$ can be extended to a factor $f(x, y)$ of $A_{\mathrm{K}}(x, y)$ and the variables of $A_{\mathrm{C}}(\bar{x}, \bar{y})$ and $f(x, y)$ satisfying the relations: $\bar{x}=x^{w}, \bar{y}^{w}=y$. In particular for some $\left(x_{0}, y_{0}\right)$ we have $\bar{x}_{0}=x_{0}^{w}, \bar{y}_{0}^{w}=y_{0}$, and $\left(x_{0}, y_{0}\right)$ is a zero point of $f(x, y)$. Obviously $x_{0} \notin\{0,1,-1\}$. From $\left(\bar{x}_{0}^{d_{1}} \bar{y}_{0}^{q_{1}}\right)^{w}=1$, we have $x_{0}^{w^{2}} d_{1} y_{0}^{q_{1}}=1$, i.e. $x_{0}^{d} y_{0}^{2}=1$.

As $f(x, y)$ is a factor in $A_{\mathrm{K}}(x, y)=A_{\mathrm{J}_{*}}(x, y)$, we see that the system

$$
\left\{\begin{array}{l}
A_{J_{*}}(x, y)=0 \\
x^{d} y^{2}-1=0
\end{array}\right.
$$

has a solution $\left(x_{0}, y_{0}\right)$ with $x_{0} \notin\{0,1,-1\}$. We get a contradiction with Lemma 5.5 $\diamond$ Note that $s-1$ is a cyclic surgery slope of $J_{*}$ (provided by [13, Proposition 5.4]).

Lemma 7.5. If $\left(x_{0}, y_{0}\right)$ is a solution of the system

$$
\begin{cases}A_{J_{*}}(x, y) & =0 \\ x^{s-1} y-1 & =0\end{cases}
$$

then $x_{0}$ is either 1 or -1 .

Proof. If $x_{0} \neq 1$ or -1 , it implies that there is a curve component $X_{0}$ in $X^{*}(M)$ such that $\tilde{X}_{0}$ has a point at which $\tilde{f}_{\mu^{s-1} \lambda}=0$ but $\tilde{f}_{\mu} \neq 0$. This is impossible as $\tilde{f}_{\mu^{s-1} \lambda}$ has the minimal zero degree at every point of $\tilde{X}_{0}$ (because $J_{*}$ is a small knot, $s-1$ is a cyclic surgery slope but is not a boundary slope). $\diamond$ 
Lemma 7.6. The integer $s-1$ is divisible by $w^{2}$.

Proof. The proof is similar to that of Lemma 7.4, only replacing Lemma [5.5 by Lemma 7.5, First note that $s-1$ is an odd number (as $s=-3 l_{*}\left(l_{*}+1\right.$ ) is even). So if $s-1$ is not divisible by $w^{2}$, then the reduced form $d_{1} / q_{1}$ of the rational number $(s-1) / w^{2}$ has denominator $q_{1}>2$. Now arguing as in the proof of Lemma 7.4 starting from the $d_{1} / q_{1}$-surgery on $C$, we see that the system

$$
\begin{cases}A_{\mathrm{J}_{*}}(x, y) & =0 \\ x^{s-1} y-1 & =0\end{cases}
$$

has a solution $\left(x_{0}, y_{0}\right)$ with $x_{0} \neq 1,-1$. This gives a contradiction with Lemma $7.5 \diamond$

Corollary 7.7. The winding number $w=1$.

Proof. This follows immediately from Lemmas 7.4 and $7.6 \diamond$

Lemma 7.8. The companion knot $C$ has the same half-integral toroidal slope as $J_{*}$ and $C$ is one of $k(l, m, 0, p)$ with $p$ non-positive and with $(2 p-1) \mid l$.

Proof. It follows from Corollary 7.7 and Proposition 2.8 that $A_{\mathrm{C}}(x, y)$ is a factor of $A_{\mathrm{K}}(x, y)=A_{\mathrm{J}_{*}}(x, y)$. So Proposition 5.8 says that $r$ is also a toroidal slope of $C$ and $C$ is one of $k(l, m, n, p)$. Now arguing as in the case when $K$ is hyperbolic, we see that $C$ is one of $k(l, m, 0, p)$ with $p$ non-positive and with $(2 p-1) \mid l . \diamond$

Lemma 7.9. For $C=k(l, m, 0, p)$ given by Lemma 7.8, we have $m=-1$ unless $C=k(2,2,0,0)$ or $C=k(-2, m, 0,0)$.

Proof. If $m \neq-1$, then by [12, Theorem 2.1 (d)] the $(s-1)$-surgery on $C=k(l, m, 0, p)$ is a Seifert fibred manifold whose base orbifold is a 2 -sphere with exactly three cone points, except when $C$ is one of $k(-2, m, 0, p), k(2,2,0,0), k(2,3,0,1), k(3,2,0,1), k(2,2,0,2)$. As we knew that $p$ is non-positive and $2 p-1$ divides $l$, these exceptional cases can be excluded except $k(2,2,0,0)$ or $k(-2, m, 0,0)$. So we just need to deal with the case when the $(s-1)$-surgery on $C$ is a Seifert fibred manifold whose base orbifold is a 2 -sphere with exactly three cone points. Note that $s-1$ is odd. Hence by Lemma 5.13, the system

$$
\begin{cases}A_{\mathrm{C}}(x, y) & =0 \\ x^{s-1} y-1 & =0\end{cases}
$$

has a solution $\left(x_{0}, y_{0}\right)$ with $x_{0} \neq 1,-1$. As $A_{\mathrm{C}}(x, y)$ is a factor of $A_{\mathrm{J}_{*}}(x, y)$, the point $\left(x_{0}, y_{0}\right)$ is also a solution of the system

$$
\begin{cases}A_{\mathrm{J}_{*}}(x, y) & =0 \\ x^{s-1} y-1 & =0\end{cases}
$$

which yields a contradiction with Lemma 7.5. $\diamond$

\section{Lemma 7.10.}

$$
|s-2| \leq 4 g(C) .
$$

Proof. Recall that $C$ is one of $k(l,-1,0, p), p$ non-positive, $(2,2,0,0), k(-2, m, 0,0)$, and has the same $r$ slope as $J_{*}$ and thus has the same $s$ slope as $J_{*}$. From (91) and (10) we have that for $k(l,-1,0, p), p$ non-positive, its $s$ slope and genus $g$ are given by

$$
s=-3 l(l+1)+p(-3 l-1)^{2}, g= \begin{cases}\frac{-p(3 l+1) 3 l}{2}+l^{2}+\frac{l(l-1)}{2}, & \text { if } l>0, \\ \frac{-p(-3 l-1)(-3 l-2)}{2}+l^{2}+\frac{l(l+5)}{2}+l+2, & \text { if } l<0 ;\end{cases}
$$

for $k(2,2,0,0)$ its $s$ and $g$ values are

$$
s=-18, g=5
$$

and for $k(-2, m, 0,0)$,

$$
s=-2(2 m-1)(2 m+1), g= \begin{cases}4 m^{2}-3 m, & \text { if } m>0 \\ 4 m^{2}+3 m, & \text { if } m<0 .\end{cases}
$$

In each case, one can check directly that $|s-2| \leq 4 g$ holds, being aware of some forbidden values on $l, m, p$ given by (8). 
As noted in the proof of Theorem 1.1. when the winding number $w=1$, the pattern knot $P$ is a nontrivial knot. We also have, by Lemma 2.7 that $A_{\mathrm{P}}(x, y)$ is a factor of $A_{\mathrm{K}}(x, y)=A_{\mathrm{J}_{*}}(x, y)$. Combining this fact with (3) and Lemma 7.1 we know that $P$ cannot be a torus knot. So $P$ is either a hyperbolic knot or a satellite knot.

If $P$ is a hyperbolic knot, then arguing as in the proofs of Lemmas 7.87 .9 and 7.10 we have that $P$ has the same half-integral toroidal slope as $J_{*}$, that $P$ is one of $k\left(l^{\prime},-1,0, p^{\prime}\right)$ with $p^{\prime}$ non-positive or $k(2,2,0,0)$ or $k\left(-2, m^{\prime}, 0,0\right)$, and that $|s-2| \leq 4 g(P)$.

Now from

$$
\Delta_{J_{*}}(t)=\Delta_{\mathrm{K}}(t)=\Delta_{\mathrm{C}}(t) \Delta_{\mathrm{P}}(t)
$$

we have that the genus of the given satellite knot $K$ (which is equal to that of $J_{*}$ ) is equal to the sum of the genus of $C$ and the genus of $P$. So the genus of one of $C$ and $P$, say $C$ (same argument for $P$ ), is less than or equal to the half of the genus of $J_{*}$, i.e.

$$
g(C) \leq \frac{1}{2} g\left(J_{*}\right) .
$$

So

$$
|s-2| \leq 2 g\left(J_{*}\right) \text {. }
$$

But $s=-3 l_{*}\left(l_{*}+1\right)$ and $g\left(J_{*}\right)=l_{*}^{2}+\frac{l_{*}\left(l_{*}-1\right)}{2}$, which do not fit in the inequality (28). This contradiction shows that $P$ cannot be hyperbolic.

So $P$ is a satellite knot. Let $\left(C_{1}, P_{1}\right)$ be a pair companion knot and pattern knot for $P$. Once again as $P$ is fibred, each of $C_{1}$ and $P_{1}$ is fibred, and the winding number $w_{1}$ of $P_{1}$ with respect to $C_{1}$ is larger than zero. Making use of the fact that $A_{\mathrm{P}}(x, y) \mid A_{\mathrm{J}_{*}}(x, y)$, one can show, similarly as for the pair $(C, P)$, that $C_{1}$ can be assumed to be hyperbolic, that $w_{1}=1$, that $C_{1}$ has the same $r$ and $s$ values as $J_{*}$, that $C_{1}=k\left(l^{\prime \prime},-1,0, p^{\prime \prime}\right)$ for some non-positive $p^{\prime \prime}$ or $k(2,2,0,0)$ or $k\left(-2, m^{\prime \prime}, 0,0\right)$, and that $|s-2| \leq 4 g\left(C_{1}\right)$. Now from the equality

$$
\Delta_{J_{*}}(t)=\Delta_{\mathrm{K}}(t)=\Delta_{\mathrm{C}}(t) \Delta_{\mathrm{C}_{1}}(t) \Delta_{\mathrm{P}_{1}}(t)
$$

we see that one of $g(C)$ and $g\left(C_{1}\right)$ is less than or equal to $\frac{1}{2} g\left(J_{*}\right)$. This leads to a contradiction just as in the preceding paragraph. So $P$ cannot be a satellite knot, and this final contradiction completes the proof of Theorem 1.2 .

\section{References}

[1] K. Baker, Closed essential surfaces in the complements of large volume Berge knots, preprint (2005), arXiv:math/0509082.

[2] J. Berge, Some knots with surgeries yielding lens spaces, unpublished draft.

[3] S. Boyer, X. Zhang, Every nontrivial knot in the 3-sphere has nontrivial A-polynomial, Proc. Amer. Math. Soc. 133 (2005) 2813-2815.

[4] S. Boyer, X. Zhang, A proof of the finite filling conjceture, J. Differential Geometry 59 (2001) $87-176$.

[5] S. Boyer, X. Zhang, Cyclic surgery and boundary slopes, AMS/IP Studies in Advanced Mathematics 2 (1997), 62-79.

[6] S. Boyer, X. Zhang, On Culler-Shalen seminorms and Dehn filling, Ann. of Math., 248 (1998) 737-801.

[7] G. Burde, H. Zieschang, Knots, Second edition. de Gruyter Studies in Mathematics, 5. Walter de Gruyter \& Co., Berlin, 2003. xii+559 pp. 
[8] D. Cooper, M. Culler, H. Gillet, D. Long, P. Shalen, Plane curves associated to character varieties of 3-manifolds, Invent. Math. 118 (1994), 47-84.

[9] M. Culler, C. M. Gordon, J. Luecke, P. Shalen, Dehn surgery on knots, Ann. of Math. 125 (1987) 237-300.

[10] M. Culler, P. Shalen, Varieties of group representations and splittings of 3-manifolds, Ann. of Math. 117 (1983) 109-146.

[11] N. Dunfield, S. Garoufalidis, Nontriviality of the A-polynomial for knots in $S^{3}$, Algebr. Geom. Topol. 4 (2004) 1145-1153.

[12] M. Eudave-Muñoz, Non-hyperbolic manifolds obtained by Dehn surgery on hyperbolic knots, Geometric topology (Athens, GA, 1993) 35-61.

[13] M. Eudave-Muñoz, On hyperbolic knots with Seifert fibered Dehn surgeries Proceedings of the First Joint Japan-Mexico Meeting in Topology (Morelia, 1999). Topology Appl. 121 (2002), no. 1-2, 119-141.

[14] P. Ghiggini, Knot Floer homology detects genus-one fibred knots, Amer. J. Math. 130 (2008), no. 5, 1151-1169.

[15] C. Gordon, Dehn surgery and satellite knots, Trans. Amer. Math. Soc. 275 (1983), 687-708.

[16] C. Gordon, J. Luecke, Only integral Dehn surgeries can yield reducible manifolds, Math. Proc. Camb. Phil. Sot. 102 (1987), 97-101.

[17] C. Gordon, J. Luecke, Non-integral toroidal Dehn surgeries, Comm. Anal. Geom. 12 (2004), $417-485$.

[18] M. Hedden, On knot Floer homology and cabling, II, Int. Math. Res. Not. IMRN 2009, no. 12, $2248-2274$.

[19] M. Hirasawa, K. Murasugi, D. Silver, When does a satellite knot fiber?, Hiroshima Journal of Mathematics, 38 (2008), 411-423.

[20] P. Kronheimer, T. Mrowka, Khovanov homology is an unknot-detector, Publ. Math. Inst. Hautes Études Sci. 113 (2011), no. 1, 97-208.

[21] P. Kronheimer, T. Mrowka, Dehn surgery, the fundamental group and SU(2), Math. Research Letter 11 (2004) 741-754.

[22] S. Lang, Algebra, Third Edition, Addison Wesley Publishing Company 1993.

[23] Y. Ni, Knot Floer homology detects fibred knots, Invent. Math. 170 (2007), no. 3, 577-608.

[24] P. Ozsváth, Z. Szabó, Holomorphic disks and knot invariants, Adv. Math. 186 (2004), no. 1, 58-116.

[25] P. Ozsváth, Z. Szabó, Holomorphic disks and genus bounds, Geom. Topol. 8 (2004), 311-334.

[26] P. Shanahan, Cyclic surgery and the A-polynomial, Topology Appl., 108 (2000), 7-36.

[27] T. Soma, On preimage knots in $S^{3}$, Proc. Amer. Math. Sco. 100 (1987), 589-592.

[28] X. Zhang, The C-polynomial of a knot, Topology Appl. 139 (2004), 185-198. 